\title{
Radio emission models of colliding-wind binary systems. Inclusion of IC cooling
}

\author{
J. M. Pittard ${ }^{1}$, S. M. Dougherty ${ }^{2}$, R. F. Coker ${ }^{3}$, E. O'Connor ${ }^{2,4}$, and N. J. Bolingbroke ${ }^{2,5}$ \\ ${ }^{1}$ School of Physics and Astronomy, The University of Leeds, Woodhouse Lane, Leeds LS2 9JT, UK \\ e-mail: jmp@ast.leeds.ac.uk \\ 2 National Research Council of Canada, Herzberg Institute for Astrophysics, Dominion Radio Astrophysical Observatory, PO Box 248, \\ Penticton, BC, V2A 6J9, Canada \\ 3 Los Alamos National Laboratory, X-2 MS T-087, Los Alamos, NM 87545, USA \\ ${ }^{4}$ Physics Department, University of Prince Edward Island, Charlottetown, PEI, Canada \\ 5 Department of Physics and Astronomy, University of Victoria, 3800 Finnerty Rd, Victoria, BC, Canada
}

Received 17 June 2005 / Accepted 3 October 2005

ABSTRACT

Radio emission models of colliding wind binaries (CWBs) have been discussed by Dougherty et al. (2003). We extend these models by considering the temporal and spatial evolution of the energy distribution of relativistic electrons as they advect downstream from their shock acceleration site. The energy spectrum evolves significantly due to the strength of inverse-Compton (IC) cooling in these systems, and a full numerical evaluation of the synchrotron emission and absorption coefficients is made. We have demonstrated that the geometry of the WCR and the streamlines of the flow within it lead to a spatially dependent break frequency in the synchrotron emission. We therefore do not observe a single, sharp break in the synchrotron spectrum integrated over the WCR, but rather a steepening of the synchrotron spectrum towards higher frequencies. We also observe that emission from the wind-collision region (WCR) may appear brightest near the shocks, since the impact of IC cooling on the non-thermal electron distribution is greatest near the contact discontinuity (CD), and demonstrate that the impact of IC cooling on the observed radio emission increases significantly with decreasing binary separation. We study how the synchrotron emission changes in response to departures from equipartition, and investigate how the thermal flux from the WCR varies with binary separation. Since the emission from the WCR is optically thin, we see a substantial fraction of this emission at certain viewing angles, and we show that the thermal emission from a CWB can mimic a thermal plus non-thermal composite spectrum if the thermal emission from the WCR becomes comparable to that from the unshocked winds. We demonstrate that the observed synchrotron emission depends upon the viewing angle and the wind-momentum ratio, and find that the observed synchrotron emission decreases as the viewing angle moves through the WCR from the WR shock to the O shock. We obtain a number of insights relevant to models of closer systems such as WR 140. Finally, we apply our new models to the very wide system WR 147. The acceleration of non-thermal electrons appears to be very efficient in our models of WR 147, and we suggest that the shock structure may be modified by feedback from the accelerated particles.

Key words. stars: binaries: general - stars: early-type - stars: individual: WR 147 - stars: Wolf-Rayet - stars: winds, outflows radio continuum: stars

\section{Introduction}

Radio observations of early-type stars reveal that they can be sources of both thermal and non-thermal radiation. The thermal emission is readily explained as free-free emission within the stellar wind (Wright \& Barlow 1975), while the nonthermal emission is attributed to relativistic electrons moving in a magnetic field. The consensus is that the relativistic electrons in the winds of massive stars are created through diffusive shock acceleration (DSA), though other mechanisms have been investigated (e.g., Jardine et al. 1996). Observations of the WR+OB binary systems WR 140 (Dougherty et al. 2005), WR 146 (Dougherty et al. 1996, 2000) and WR 147
(Moran et al. 1989; Churchwell et al. 1992; Williams et al. 1997; Niemela et al. 1998) clearly show the non-thermal emission in these binaries arises from a wind-collision region (WCR) bounded by strong shocks formed where the two stellar winds collide. There is strong evidence that all WR stars that exhibit non-thermal emission are binary (Dougherty \& Williams 2000).

For synchrotron emission to be observed, the relativistic electrons must be within the optically thin region of the stellar wind(s), which in the case of massive stars implies at more than a few hundred stellar radii from the underlying star. These large radii are problematic for single star models of synchrotron 
emission, where the declining velocity jump and compression ratio with increasing radius of wind-embedded shocks produces a rapidly decreasing synchrotron emissivity (Van Loo 2005).

CWB systems are important objects for investigating the underlying physics of DSA because they provide access to higher mass, radiation and magnetic field energy densities than is possible through studies of supernova remnants. However, until the work of Dougherty et al. (2003, hereafter Paper I), CWB models of radio spectra were typically based on a highly simplified model consisting of a thermal source plus a pointlike source of non-thermal emission attenuated by free-free absorption (e.g., Chapman et al. 1999; Monnier et al. 2002). Use of these models often resulted in difficulties in fitting observational data (see, e.g., Williams et al. 1990; White \& Becker 1995). As a first step toward the construction of more realistic models with better predictive qualities, a hydrodynamical model of the WCR was used in Paper I to obtain a more accurate representation of the spatial distribution of the free-free and non-thermal emission from CWB systems. Several important scaling laws were determined and, in spite of other simplifying assumptions, the results of that work showed great promise in comparisons with observed continuum spectra and images. We refer the reader to Paper I for essential background to the work presented here.

Inverse-Compton (hereafter IC) cooling is an important energy-loss mechanism for relativistic electrons in CWB systems, since the energy distribution will evolve as the electrons advect away from the shocks. The inclusion of this process and the calculation of emission and absorption coefficients from an arbitrary energy distribution of non-thermal electrons are the main enhancements to the model and the work presented in this paper. Following examination of the effect of these mechanisms, we apply these new models to WR 147, and close by describing future directions for this work.

\section{Modelling the radio emission from CWBs}

In Paper I, the radio emission and absorption from a CWB system was calculated from a 2D axis-symmetric hydrodynamical model of the stellar winds and the collision zone. The temperature and density values on the hydrodynamic grid were used to calculate the free-free emission and absorption coefficients from each grid cell. The synchrotron emission and selfabsorption from each cell within the WCR was calculated assuming that the distribution of relativistic electrons in each cell could be specified by a power-law, i.e. $n_{\mathrm{e}}(\gamma) \propto \gamma^{-p}$, where $\gamma$ is the Lorentz factor ${ }^{1}$. The synchrotron emissivity, $P_{v}$, also followed a power-law $\left(P_{v} \propto v^{-(p-1) / 2}\right)$, which was assumed to be valid over all frequency, $v$. The flux and intensity distribution at a specified frequency were then obtained from a radiative transfer calculation.

The hydrodynamical simulations of Paper I do not provide direct information on the magnetic field or relativistic particle distribution, so the magnetic energy density $U_{B}$, and the

\footnotetext{
${ }^{1}$ For a strong shock with a compression ratio of 4 , test particle theory predicts that $p=2$ (see references in Paper I).
}

relativistic electron energy density $U_{\text {rel }}$, were set proportional to the thermal particle internal energy density, $U_{\mathrm{th}}$ :

$U_{B}=B^{2} / 8 \pi=\zeta_{B} U_{\mathrm{th}}$,

and

$U_{\text {rel }}=\int n_{\mathrm{e}}(\gamma) \gamma m_{\mathrm{e}} c^{2} \mathrm{~d} \gamma=\zeta_{\text {rel }} U_{\text {th }}$.

$U_{\text {th }}=\frac{P}{\Gamma-1}$, with $P$ the gas pressure and $\Gamma$ the adiabatic index, assumed to be $5 / 3$, as for an ideal gas. $\zeta_{B}$ and $\zeta_{\text {rel }}$ are constants which determine the amount of synchrotron emission and absorption, and the Razin turnover frequency in our models. Since we did not attempt to model the acceleration of the relativistic particles from basic principles, the values of $\zeta_{B}$ and $\zeta_{\text {rel }}$ are not determined in a self-consistent fashion. Instead, when modelling specific systems, their values are chosen to best match the observed radio emission. Our previous models in Paper I showed that if $\zeta_{B}=\zeta_{\text {rel }}$, then values of around $1 \%$ are required (see also Mioduszewski et al. 2001). Unless otherwise noted, $\zeta_{B}=\zeta_{\text {rel }}=\zeta$, though we relax this restriction in Sect. 3.6 and in some models in Sect. 4.

\subsection{The hydrodynamical code}

In contrast to Paper I, a hydrodynamical code with adaptive mesh refinement is used here (Falle \& Komissarov 1996; Falle et al. 1998). Such codes concentrate more cells where the flow is interesting, for example near shocks, and fewer cells where it is uniform, resulting in significantly shorter computational times. We use a small amount of artificial viscosity in the calculations to prevent the development of the carbuncle instability at the apex of the WCR. This numerical artifact develops when shocks are locally aligned with the grid (Walder 1994; Leveque 1998).

\subsection{The non-thermal electron energy spectrum}

In this work, it is assumed that the acceleration of relativistic electrons at the shocks bounding the WCR produces an energy distribution with a power-law spectrum. When particle acceleration is relatively inefficient, this is only strictly true in the absence of cooling. However, the resulting deviation from a pure power-law is restricted to energies which are very near to the high energy cutoff (Chen \& White 1991; Van Loo et al. 2005), and we ignore this effect in the current work. In contrast, when DSA is efficient, non-linear effects modify the pre-shock wind speeds over a length scale comparable to the diffusion length of the energetic particles, and slow them before they undergo a shock transition. Thus higher energy particles with larger diffusion length scatter in regions where the pre-shock wind speed is closer to the terminal speed of the stellar wind, while low energy particles do not diffuse very far from the subshock and scatter in regions where the wind speed is lower due to its journey through much of the shock precursor. This speed profile results in a concave upward curvature to the non-thermal particle spectrum, where $p$ decreases with increasing energy (Ellison et al. 2004, and references therein). Efficient particle 

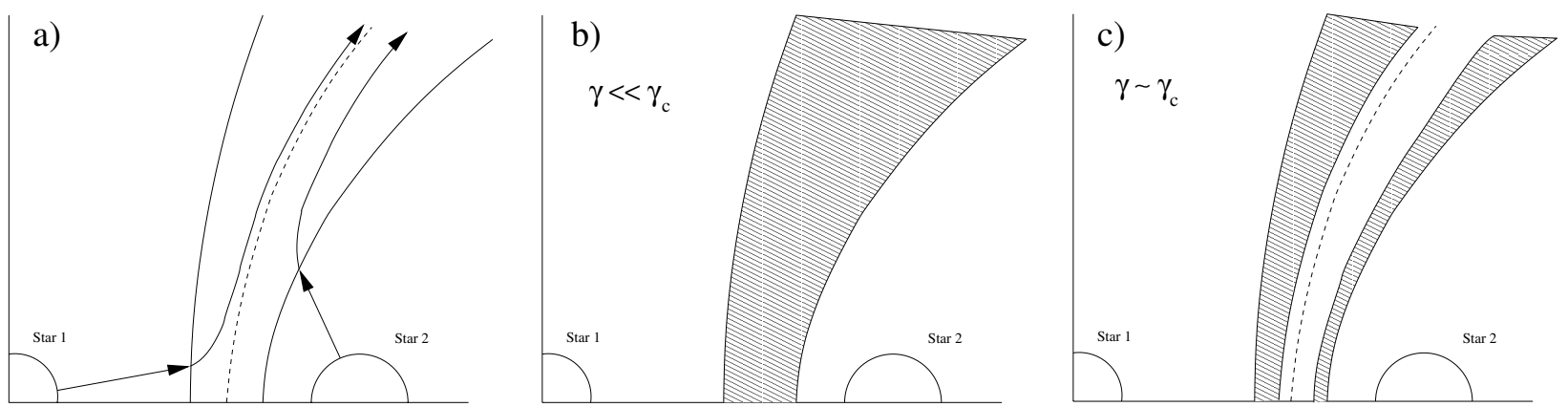

Fig. 1. Schematic diagrams of the flow within the WCR and the distribution of relativistic electrons. a) The flow within the winds and the WCR, which is located nearer the star with the weaker wind (star 2 in this example). The dotted line depicts the contact discontinuity (CD) between the two winds. Either side of the CD is a shock which separates the high Mach number wind from high pressure post-shock gas. Example streamlines are shown for material leaving the stars along specific direction vectors. b) The distribution of relativistic electrons with Lorentz factor $\gamma \ll \gamma_{\mathrm{c}}$, assuming that such electrons are first-order Fermi accelerated at the shocks. c) As b) but for $\gamma \sim \gamma_{\mathrm{c}}$. IC cooling effectively excludes electrons with Lorentz factor $\gamma_{\mathrm{c}}$ from a region near the CD.

acceleration can also flatten the non-thermal particle energy distribution i.e. $p<2$. Modelling this process is non-trivial and we make no attempt in the current work to calculate these effects self-consistently. However, we discuss it further and perform calculations with a non-thermal particle spectrum where $p<2$ in Sect. 4.

Relativistic electrons that scatter out of the shock with Lorentz factor $\gamma \lesssim 10^{5}$ are frozen into the post-shock flow (White 1985). As they are advected out of the system, the relativistic electrons lose energy through the action of several processes, including ionic cooling, magneto-bremsstrahlung cooling, and IC cooling. Since IC cooling strongly dominates magneto-bremsstrahlung cooling in this work (see Eq. (14) of Paper I), we do not consider the latter here. Cooling reduces the non-thermal electron energy density at a given $\gamma$ and alters the energy spectrum of these electrons from a simple power-law. Thus, in this work we assume the non-thermal electron energy spectrum is a power-law at the shocks, but let it evolve as the electrons advect downstream from the shocks. As noted earlier, the inclusion of these processes is the main enhancement to the model since Paper I, and in the following sub-sections we describe in detail our method of implementation.

\subsubsection{Inverse Compton cooling}

The high UV luminosity of the stars in CWBs means that IC cooling of the relativistic electrons in the WCR can be very efficient, particularly for high $\gamma$ electrons. In the Thomson limit, the rate of energy loss of a relativistic electron exposed to an isotropic distribution of photons by IC scattering is (Rybicki \& Lightman 1979)

$P_{\text {compt }}=\frac{4}{3} \sigma_{\mathrm{T}} c \gamma^{2} \beta^{2} U_{\mathrm{ph}}$,

where $\sigma_{\mathrm{T}}$ is the Thomson cross section, $\beta=v / c=\sqrt{1-1 / \gamma^{2}}$, $U_{\mathrm{ph}}$ is the energy density of photons, and where elastic scattering is assumed and quantum effects on the cross section are neglected. For a single star and relativistic particles with $\beta \approx 1$, we can rewrite Eq. (3) as

$$
\left.\frac{\mathrm{d} \gamma}{\mathrm{d} t}\right|_{\mathrm{i} c}=\frac{\sigma_{\mathrm{T}} \gamma^{2}}{3 \pi m_{\mathrm{e}} c^{2}} \frac{L}{r^{2}}=8.61 \times 10^{-20} \gamma^{2} f \mathrm{~s}^{-1},
$$

where $L$ is the stellar luminosity, $r$ is the distance of the particles from the star, and $f=\frac{L}{r^{2}}\left(\mathrm{erg} \mathrm{s}^{-1} \mathrm{~cm}^{-2}\right)$ is a measure of the radiative flux on an electron at distance $r$. For a given electron, the reduction in $\gamma$ due to IC cooling can be calculated if the integral of $f t$ along its streamline is known. In a CWB, $f=\frac{L_{0}}{r_{\mathrm{O}}^{2}}+\frac{L_{\mathrm{WR}}}{r_{\mathrm{WR}}^{2}}$ due to the radiation field of both stars. The value of $f t$ in any given cell on the hydrodynamic grid can be obtained by the specification of a suitable scalar in the code. It is then a simple matter during post-processing to calculate the IC-cooled relativistic energy spectrum at that location.

The influence of IC cooling increases in magnitude as plasma flows from the shocks toward the contact discontinuity - this reflects the increased exposure to the high intensity stellar radiation field from the time when the relativistic electrons were accelerated in one of the shocks bounding the WCR. In addition, since the rate of cooling is proportional to $\gamma^{2}$, IC cooling is most significant for electrons with the highest energies. We can therefore define a characteristic Lorentz factor, $\gamma_{\mathrm{c}}$, where IC cooling becomes significant for flow near the apex of the WCR. The flow morphology and the distribution of electrons with a specific Lorentz factor is shown in Fig. 1. For low-energy electrons $\left(\gamma \ll \gamma_{\mathrm{c}}\right)$, IC cooling is negligible and such electrons may exist throughout the WCR, as shown in Fig. 1b. In contrast, IC cooling is significant for electrons with $\gamma \gtrsim \gamma_{\mathrm{c}}$, which rapidly lose energy as they flow away from the shocks. In such circumstances, electrons with $\gamma \sim \gamma_{\mathrm{c}}$ are effectively confined to narrow regions near the shocks, and absent from the central volume of the WCR, as illustrated in Fig. 1c.

\subsubsection{Ionic (coulomb) cooling}

Relativistic electrons may also lose energy through Coulomb collisions with thermal ions. The rate of energy loss through 
this process for electrons of energy $\gamma$ is (cf. Chen \& White 1991)

$$
\left.\frac{\mathrm{d} \gamma}{\mathrm{d} t}\right|_{\text {coul }}=\frac{4 \pi e^{4} n_{\mathrm{i}} \ln \Lambda}{m_{\mathrm{e}}^{2} c^{3} \sqrt{\gamma^{2}-1}}=3.0 \times 10^{-14} \frac{n_{\mathrm{i}} \ln \Lambda}{\sqrt{\gamma^{2}-1}} \mathrm{~s}^{-1},
$$

where $\ln \Lambda=29.7+\ln n_{\mathrm{i}}^{-1 / 2} T_{6}$ is the Coulomb logarithm (e.g., Cowie \& McKee 1977) ${ }^{2}, T_{6}$ is the temperature in units of $10^{6} \mathrm{~K}$, and $n_{\mathrm{i}}$ is the number density of ions $\left(\mathrm{cm}^{-3}\right)$. As for IC cooling, we can estimate the degree of ionic cooling, namely by constructing a scalar to track the value of $n_{\mathrm{i}} t \ln \Lambda$ in each hydrodynamic cell of hot shocked gas. Ionic coooling is greatest for non-thermal electrons with relatively low energies, and while it is included in our model we note that there is no observational signature of this process on the synchrotron spectrum above $10 \mathrm{MHz}$ (see Fig. 3). However, we include it for completeness.

\subsubsection{Maximum energy of relativistic electrons}

In the standard DSA scheme, particles are assumed to scatter from MHD waves in the background plasma, either preexisting or generated by the counter-streaming ions themselves (Bell 1978). There is strong evidence that the efficiency of particle acceleration in the DSA scheme is dependent on the orientation of the upstream magnetic field to the shock normal (see the review by Blandford \& Eichler 1987). When the shock is quasi-perpendicular the competing "shock drift" mechanism may be more efficient (Jokipii 1987), though the acceleration rates become equal in shocks with a high level of magnetic turbulence since these have no well-defined obliquity ${ }^{3}$.

Since the physics of electron injection is not yet fully understood, we ignore these complications in the present work, and assume that both the normalization of the non-thermal particle spectrum, and the maximum energy that the accelerated particles can reach, are independent of spatial position. To estimate the value of $\gamma_{\max }$, we balance the rate of energy gain through 1st-order Fermi acceleration, by the rate of energy loss due to IC cooling (the dominant cooling mechanism for relativistic electrons with large $\gamma$ ). The 1 st-order Fermi power is (Chen \& White 1991; Van Loo et al. 2005) ${ }^{4}$

$$
\left.\frac{\mathrm{d} \gamma}{\mathrm{d} t}\right|_{\mathrm{acc}}=\frac{4}{3} \frac{\chi_{\mathrm{c}}-1}{\chi_{\mathrm{c}}} \frac{u \gamma \beta^{2}}{\lambda} \frac{2 u}{c},
$$

where $\chi_{\mathrm{c}}$ is the ratio of upstream to downstream flow speeds, $u$ is the shock velocity, $c$ is the speed of light, and $\lambda$ is the meanfree path of the accelerating charge. This latter quantity is somewhat uncertain, but is typically estimated as several gyroradii. We adopt $\lambda \approx 3 r_{\mathrm{g}}$, where the gyro-radius $r_{\mathrm{g}}=p c / q B$

\footnotetext{
2 While we use this expression for the Coulomb logarithm, Li (2001) note that it should be reduced to about half of its conventional value.

3 The obliquity of the shocks expected in CWB systems is described by Eichler \& Usov (1993).

${ }^{4}$ Chen \& White (1991) erroneously assume that shock-accelerated electrons return to the shock after one scattering, whereas electrons are actually scattered $c / 2 u$ times before recrossing the shock (Van Loo et al. 2005).
}

(White 1985) ${ }^{5}$, and $p$ and $q$ are the momentum and charge of the accelerating particle. Assuming that we are in the strong limit for adiabatic shocks $\left(\chi_{\mathrm{c}}=4\right)$ and that the accelerated charge is a highly relativistic electron $(\beta=1, \gamma \gg 1)$, we have

$$
\left.\frac{\mathrm{d} \gamma}{\mathrm{d} t}\right|_{\mathrm{acc}}=\frac{1}{3} \frac{u q B}{m_{\mathrm{e}} c^{2}} \frac{2 u}{c} .
$$

By balancing Eqs. (4) with (7) we obtain

$$
\gamma_{\max } \approx 400 u r_{\mathrm{O}} \sqrt{\frac{B}{L_{\mathrm{O}}}}
$$

Equation (8) is equivalent to Eq. (14) in Eichler \& Usov (1993). Due to its dependence on $B, \gamma_{\max } \propto \zeta_{B}^{1 / 4}$. In our standard model, $\gamma_{\max } \approx 7 \times 10^{4}$ at the point where the shocks intersect the line of centers through the stars. However, since the value of $\zeta$ required to match observational data is not known a priori, in all of the work presented in this paper we fix $\gamma_{\max }=10^{5}$, which is also consistent with the maximum $\gamma$ at which relativistic electrons are frozen into the post-shock flow.

\subsection{NT emission and absorption}

With a power-law energy distribution, the magnetobremsstrahlung emission can be calculated analytically, as in Paper I. However, given an arbitrary energy distribution of nonthermal electrons, calculation of the magneto-bremsstrahlung emission from the downstream flow requires a numerical integration of the magneto-bremsstrahlung emissivity over the full energy range of the relativistic electron energy spectrum.

\subsubsection{Synchrotron emission}

In the synchrotron limit, the emission arising from a single relativistic electron with Lorentz factor $\gamma$ is

$P(v)=\frac{\sqrt{3} q^{3} \beta^{2} B \sin \alpha}{m_{\mathrm{e}} c^{2}} F\left(v / v_{c}\right)$,

where $\alpha$ is the pitch angle of the particle relative to the direction of the $B$-field, and $F\left(v / v_{c}\right)$ is a dimensionless function describing the total power spectrum of the synchrotron emission, with $v_{c}$ the frequency where the spectrum cuts off, given by

$v_{c}=\frac{3 \gamma^{2} q B \sin \alpha}{4 \pi m_{\mathrm{e}} c}=\frac{3}{2} \gamma^{2} \nu_{\mathrm{b}} \sin \alpha$.

$\nu_{\mathrm{b}}=\omega_{\mathrm{b}} / 2 \pi$, where $\omega_{\mathrm{b}}=q B / m_{\mathrm{e}} c$ is the cyclotron frequency of the electron. $\omega_{0}=\omega_{\mathrm{b}} / \gamma$ is the orbital or relativistic gyration frequency of the electron. Values for $F\left(v / v_{c}\right)$ are tabulated in Ginzburg \& Syrovatskii (1965). Throughout the remainder of this paper we assume that $\sin \alpha=1$. While this means that our calculations will slightly overestimate the synchrotron emission for a given $\zeta_{B}$, a more detailed consideration is not warranted at the present time. At the stagnation point in our "standard model", $v_{c}=260 \mathrm{MHz}$ for $\gamma=100$, and $26 \mathrm{GHz}$ for $\gamma=10^{4}$.

5 This is equivalent to specifying that the diffusion coefficient is proportional to the particle energy. 
The synchrotron emissivity formula (Eq. (9)) may be used even for mildly relativistic electrons (e.g., $\gamma<2$ ), provided that $\chi \gtrsim 100$, where

$\chi=\frac{v}{v_{\mathrm{b}}}=\frac{\omega}{\omega_{\mathrm{b}}}=\frac{\omega}{\gamma \omega_{0}}$

(see Fig. 2 of Mahadevan et al. 1996). Since $\chi \propto v / B, \chi$ is small when we have low frequencies and high $B$-fields (or, alternatively when the binary separation is small, since $B \propto 1 / D_{\text {sep }}$ ). The lowest frequency we examine is $200 \mathrm{MHz}$. In our "standard model" (see Sect. 3.1), $v_{\mathrm{b}}=1.7 \times 10^{4} \mathrm{~Hz}$ and $B=5.9 \mathrm{mG}$ at the stagnation point of the WCR, which yields $\chi=1.2 \times 10^{4}$ when $v=200 \mathrm{MHz}$. The declining $B$-field means that $v_{\mathrm{b}}$ declines with off-axis distance, which leads to higher values of $\chi$. In the most extreme case which we consider $\left(D_{\text {sep }}=2 \times 10^{14} \mathrm{~cm}\right.$, $\left.\zeta_{B}=10^{-2}\right), \chi>100$ throughout the WCR, justifying our use of the synchrotron emissivity formula.

\subsubsection{Synchrotron self-absorption}

Synchrotron self-absorption (SSA) or, more accurately at low $\gamma$, magneto-bremsstrahlung self-absorption, is intimately linked to the energy spectrum of the non-thermal particles and their magneto-bremsstrahlung emissivity, and in Appendix A we show how the calculation of SSA is performed. The SSA turnover frequency for a source subtending a solid angle $\Omega$ (steradians) is given by (for $p=2$; cf. Pacholczyk 1970)

$v_{\mathrm{SSA}}=46.3 B^{1 / 6} S_{v}^{1 / 3} v^{1 / 6} \Omega^{-1 / 3} \mathrm{~Hz}$,

where $B$ is in Gauss, and $S_{v}$ is the synchrotron flux in mJy at any frequency $v$ (in $\mathrm{Hz}$ ) where the source is optically thin. For our "standard" CWB model (without IC cooling) with $\zeta=$ $10^{-4}$ (see Sect. 3.1), the magnetic field strength at the apex of the WCR is $5.9 \mathrm{mG}, S_{v}=0.026 \mathrm{mJy}$ at $v=81.1 \mathrm{GHz}$, and $\Omega \sim 3 \times 10^{-15}$ ster (the spatial extent of synchrotron emission from the WCR is $\sim 60 \times 5$ mas), giving $v_{\mathrm{SSA}} \approx 20 \mathrm{MHz}^{6}$. For wide CWBs, SSA is negligible at $\mathrm{GHz}$ frequencies, but may become important in closer systems.

\subsubsection{The Razin effect}

When relativistic charges are surrounded by a plasma (as opposed to existing in a vacuum), the beaming effect that characterizes synchrotron radiation is suppressed. As noted in Paper I, the refractive index of the medium reduces the Lorentz factor of the electron to

$\gamma^{\prime}=\frac{\gamma}{\sqrt{1+\gamma^{2} v_{0}^{2} / v^{2}}}$,

${ }^{6}$ Note that the calculations in Paper I greatly overestimate the strength of SSA (see, e.g., Fig. 4 in that paper). This error resulted from the use of Eq. (6.53) in Rybicki \& Lightman (1979) that takes $C$ from Eq. (6.20a). We had assumed it was taken from Eq. (6.20b), since this is the case for Eq. (6.36). This led to the erroneous conclusion in Paper I that the SSA turnover frequency and the characteristic cutoff frequency from the Razin effect are comparable in our "standard" CWB model when $\zeta=10^{-3}$. We now find that this is the case when $\zeta \sim 10^{-2}-$ see also the discussion in Sect. 3.5. where the plasma frequency $v_{0}=\sqrt{q^{2} n_{\mathrm{e}} / \pi m_{\mathrm{e}}}$. In Paper I, this effect was approximated as an exponential reduction in flux at the characteristic cut-off frequency,

$\nu_{\mathrm{R}}=20 \frac{n_{\mathrm{e}}}{B}$.

However, since we calculate the magneto-bremsstrahlung emissivity for individual values of $\gamma$ in this work, we simply replace $\gamma$ with $\gamma^{\prime}$ in the calculations of the non-thermal emission and absorption ${ }^{7}$.

\subsection{Clumping}

There is a great deal of evidence that the winds of hot stars are clumpy (e.g., Moffat et al. 1988; Robert 1994; Lépine et al. 2000), and the effect of inhomogeneous winds on the WCR is expected to be sensitive to a number of variables (e.g., Cherepashchuk 1990; Lépine 1995). Foremost amongst these is whether the densities are low enough that the WCR and the shocked clumps are adiabatic. This is indeed the case in the wide binary systems which we consider in this paper ${ }^{8}$. In such a situation the clumps are stripped of material by a combination of dynamical processes (such as ablation) and evaporation (resulting from the conduction of heat into their interiors) as they move within the WCR.

The destruction timescale of non-radiative clumps by the action of dynamical instabilities is $t_{\mathrm{d}}=\epsilon t_{\mathrm{cc}}$ (Klein et al. 1994), where $t_{\mathrm{cc}}$ is the cloud crushing timescale, defined as $t_{\mathrm{cc}}=r_{\mathrm{c}} / v_{\mathrm{s}}$, $r_{\mathrm{c}}$ is the radius of the clump, and $v_{\mathrm{s}}$ is the shock velocity (for the stationary shocks in CWBs, $v_{\mathrm{s}}$ is equal to the pre-shock wind speed). $\epsilon \approx 3.5$ for a density ratio between the clump and the inter-clump wind of order 10-100 (Klein et al. 1994), though larger density ratios may occur in intrinsic wind shocks. A clump which passes through a shock experiences less deceleration than the inter-clump material, so a lower limit on the timescale for the clump to reach the contact discontinuity is $t_{\mathrm{cd}}=\Delta r / v_{\mathrm{s}}$, where $\Delta r$ is the distance from the shock to the CD. Clumps will be destroyed before they reach the $\mathrm{CD}$ when

$t_{\mathrm{d}} / t_{\mathrm{cd}}=\epsilon t_{\mathrm{cc}} / t_{\mathrm{cd}}=\epsilon r_{\mathrm{c}} / \Delta r<1$.

7 We note that the Razin effect is slightly more subtle than replacing $\gamma$ with $\gamma^{\prime}$ in Eqs. (9) and (10), since not all of the Lorentz factors in the function $F\left(v / v_{c}\right)$ are related to the beaming effect - see, e.g., Rybicki \& Lightman (1979), Ginzburg \& Syrovatskii (1965), or Van Loo et al. (2004) for more details. However, this has little impact on the results.

8 The interaction of a clumpy wind with a radiative WCR has been discussed by Walder \& Folini (2002). In this case the WCR may become relatively narrow, though dense, and prone to instabilities, such as nonlinear thin-shell instabilities (e.g., Stevens et al. 1992; Vishniac 1994). The fate of clumps in this situation is less certain. It is possible that they will be destroyed when they encounter the WCR, but since the post-shock gas is itself cold and dense, new "clumps" may be created as the WCR fragments under the action of these instabilities. Of note is the fact that the growth rate of the thin-shell instability depends on high tangential velocities channelling material to the extremities of the kinks (Blondin \& Marks 1996) - this channelling may be disrupted if the incident flows are clumpy, and the growth of this instability moderated to some extent, though a numerical calculation would be needed for confirmation of this idea. 
There are indications that $r_{\mathrm{c}} \sim 1 R_{*}$ at distances of $\sim 20 R_{*}$ from the star (Lépine et al. 2000; Rodrigues \& Magalhães 2000). Such clumps cannot expand faster than their sound speed, unless they behave isothermally, in which case they may expand a few times faster. The wind temperature of hot stars is $\sim 1-10 \mathrm{kK}$, and the sound speed of the clump is $\sim 10 \mathrm{~km} \mathrm{~s}^{-1}$. In WR 147, the flow time of the WR wind to the stagnation point is $\sim 10^{8} \mathrm{~s}$, so the clumps will have expanded to radii of $\sim 1000 R_{\odot}$. In our model of WR 147 in Sect. $4, \Delta r \approx 0.05 D_{\text {sep }}$, when measured along the line of centres of the stars. We thus determine that $t_{\mathrm{d}} / t_{\mathrm{cd}} \sim 0.5$, which implies that the clumps are rapidly destroyed. Material originating in the clumps is mixed in with the post-shock gas and the high sound speed of the hot WCR works to minimize the density and pressure gradients existing in the vicinity of the clump. The post-shock flow should rapidly become smooth, and the global position of the shocks and the CD should be affected very little by the clumpy nature of the winds.

If $t_{\mathrm{d}} / t_{\mathrm{cd}} \gg 1$, clumps could in theory pass completely through the WCR and into the unshocked wind of the companion star, where they would be surrounded by a bowshock and subject to a high level of ablation, before being destroyed. The possibility of clumps passing through the WCR and interacting with the photosphere of the companion star is considered by Marchenko (2002).

Another consideration is whether new clumps are created when gas in a WCR which is largely adiabatic eventually cools. We make the general statement that the growth of density perturbations within the WCR will depend on their size relative to the local cooling length of the surrounding gas (see Blondin \& Cioffi 1989), and note that any non-thermal component of the pressure (e.g., from relativistic particles) lessens the growth rate of the local thermal instability by decreasing the dependence of the total pressure on the gas pressure (e.g., Wagner et al. 2005). Since we know that there are non-thermal particles within the WCR we assume that the cold component of the WCR is smooth.

To summarize, we can distinguish the following three regions where there may or may not be clumps: stellar winds; hot WCR; cold WCR. We have demonstrated that clumps are rapidly destroyed in systems where the WCR is largely adiabatic, and have noted that clumps are less likely to be created within the WCR when there is supporting pressure from nonthermal particles. Therefore, we assume that all parts of the WCR are smooth, leaving only the nature of the stellar winds to be specified. Since the predominant effect of clumping is to introduce a scaling factor to the thermal emission, in our "standard" model in Sect. 3.1 we assume that the stellar winds are smooth. However, our models for WR 147 in Sect. 4 adopt clumpy winds in order to match the observed free-free emission with specific mass-loss rates.

\subsection{Summary of the calculations}

Our calculation of the observed radio emission from CWB systems is based on the density and temperature distribution obtained from a hydrodynamical simulation of the stellar winds and their collision. Both the stellar winds and the WCR contribute thermal emission and absorption. Free-free emission and absorption from thermal electrons is calculated as noted in Paper I, and clumping may or may not be included in the model. In addition, the WCR has emission and absorption components arising from a distribution of non-thermal electrons accelerated at the shocks and advected with the downstream flow.

To describe the non-thermal electron energy distribution we use 100, logarithmically spaced, energy bins, extending from $\gamma=1$ to $\gamma_{\max }$, and which we specify as a power-law at the shocks. The pre-cooled normalization of this energy spectrum in each cell within the WCR is obtained by setting the total non-thermal electron energy density to a specified fraction of the thermal energy density (see Eq. (2)). Scalars in the hydrodynamical code are then used to apply IC and ionic cooling, and a "cooled" non-thermal electron energy distribution is obtained in each hydrodynamical cell. As a result, the energy distribution in each cell within the WCR shows some degree of evolution away from its initial power-law (see, e.g., Fig. 3a). In the downstream flow, the energy loss from the non-thermal electrons means that $U_{\text {rel }} \leq \zeta U_{\text {th }}$. Since the magnetic energy density in each hydrodynamical cell remains tied to $U_{\text {th }}$, the magnetic field and non-thermal electron energy densities gradually diverge, with $U_{B}$ exceeding $U_{\text {rel }}$ in the downstream flow. Electrons which cool to $\gamma=1$ are assumed to return to the thermal pool.

With the non-thermal electron energy distribution and the $B$-field specified in every hydrodynamical cell within the WCR, the non-thermal emission and absorption may be calculated. If the Razin effect is specified, the de-beaming is first calculated and the effective $\gamma$ of each energy bin is reduced according to Eq. (13). The magneto-bremsstrahlung emissivity per electron is then calculated using Eq. (9). The magneto-bremsstrahlung emissivity per unit volume is obtained from multiplying the emissivity per electron by the number density of non-thermal electrons. Integration over $\gamma$ then gives the total non-thermal emission per unit volume. Synchrotron self-absorption is calculated in a similar fashion. Finally, the radiative transfer equation is solved to generate synthetic images and spectra.

\subsection{Some simplifying assumptions}

The simplifying assumptions mentioned in Sect. 2.6 of Paper I apply also to the work in this paper. An additional assumption which was not discussed previously relates to the issue of thermal cooling of hot plasma in the WCR. This is of relevance since the magnetic energy density $U_{B}$, and the energy density of relativistic electrons $U_{\text {rel }}$, are assumed to be related to the thermal energy density $U_{\text {th }}$. When the separation between the stars is sufficiently small that significant cooling of the thermal plasma takes place ( $\dot{E} \propto 1 / D_{\text {sep }}$ ), a two-fluid implementation of the post-shock flow is ideally needed.

Finally, we emphasize that in our current model the hydrodynamics of the WCR is solved in the limit that a negligible amount of energy is placed into non-thermal particles. In reality, efficient particle acceleration may greatly modify the structure of the shocks bounding the WCR and the post-shock flow. 
Our work in Sect. 4 indicates that such changes may actually take place.

\section{Parameter study}

\subsection{A standard model}

As with our initial investigations in Paper I, we examine the radio emission from a "standard" CWB model with the following parameters: $\dot{M}_{\mathrm{WR}}=2 \times 10^{-5} M_{\odot} \mathrm{yr}^{-1}, \dot{M}_{\mathrm{O}}=2 \times 10^{-6} M_{\odot} \mathrm{yr}^{-1}$, $v_{\infty, \mathrm{WR}}=v_{\infty, \mathrm{O}}=2000 \mathrm{~km} \mathrm{~s}^{-1}, D_{\text {sep }}=2 \times 10^{15} \mathrm{~cm}$. The wind momentum ratio, $\eta=\dot{M}_{\mathrm{O}} v_{\infty, \mathrm{O}} / \dot{M}_{\mathrm{WR}} v_{\infty, \mathrm{WR}}=0.1$, and the distance of the stagnation point from the WR and $\mathrm{O}$ star is respectively $r_{\mathrm{WR}}=0.76 D_{\text {sep }}$ and $r_{\mathrm{O}}=0.24 D_{\text {sep }}$. With such parameters the WCR is largely adiabatic. We assume solar abundances for the $\mathrm{O}$ star and WC-type abundances for the WR star (mass fractions $X=0, Y=0.75, Z=0.25$ ). Temperatures of $10000 \mathrm{~K}$ and an ionization structure of $\mathrm{H}^{+}, \mathrm{He}^{+}$and $\mathrm{CNO}^{2+}$ are assumed for the unshocked stellar winds. The wind temperatures are lower than those used in Paper I, but as the thermal flux from the unshocked winds is only weakly dependent on the wind temperature (through the gaunt factor), this change has only a small effect on the free-free emission (see Sect. 3.4). As before, we adopt a distance of $1.0 \mathrm{kpc}$ for our model system, and assume that $\zeta=10^{-4}$. As noted in Sect. 2.3.2, SSA is negligible at $\mathrm{GHz}$ frequencies in the standard model. However, since $B \propto \zeta^{1 / 2}$, and $S_{v} \propto \zeta^{7 / 4}$ in the optically thin limit, we find that $v_{\mathrm{SSA}} \propto \zeta^{2 / 3}$. The SSA turnover frequency is $\approx 0.2 \mathrm{GHz}$ when $\zeta=10^{-2}$.

Since IC cooling is now included in the models, appropriate luminosities for the stars must also be defined. We set $L_{\mathrm{WR}}=2 \times 10^{5} L_{\odot}$ and $L_{\mathrm{O}}=5 \times 10^{5} L_{\odot}$ for this first investigation which results in a photon energy density $U_{\mathrm{ph}}=2.3 \mathrm{erg} \mathrm{cm}^{-3}$ at the stagnation point ${ }^{9}$. For the initial non-thermal energy spectrum at the shocks, we assume a power-law energy distribution with $p=2$ and fix $\gamma_{\min }=1$ and $\gamma_{\max }=10^{5}$. This latter value is roughly the $\gamma$ at which the energy gain due to shock acceleration is balanced by the energy loss due to IC cooling, for electrons near the apex of the WCR - see Sect. 2.2.3.

All of our 2D axisymmetric hydrodynamical calculations for the standard model use a square grid with $0 \leq r \leq 4 \times$ $10^{15} \mathrm{~cm}, 0 \leq z \leq 4 \times 10^{15} \mathrm{~cm}$. The $\mathrm{z}$-axis is extended to $-4 \times$ $10^{15} \mathrm{~cm}$ with appropriate density and temperature values for the unshocked WR wind before we process the grid through our radiative transfer code. In our models, a viewing angle of $0^{\circ}$ implies lines-of-sight perpendicular to the axis of symmetry of the model.

\subsection{The thermal flux and $\tau=1$ surface of a hot star wind}

To aid the interpretation of the results from our model, we calculate the theoretical thermal flux from a single star, and the radius of optical depth unity for a sightline from infinity. As

\footnotetext{
9 We estimate $L_{\mathrm{WR}}$ and $L_{\mathrm{O}}$ from the following empirical formulae. $L_{\mathrm{WR}}$ is calculated from the relationship $\log \dot{M}=1.38 \log L-12.1$ for Galactic WC stars (Crowther 2003), while $L_{\mathrm{O}}$ is determined from $\log \dot{M}=1.69 \log L-15.4$ (Howarth \& Prinja 1989).
}

Table 1. Theoretical values of the thermal radio flux, $S_{\mathrm{ff}}$, and the radius of the $\tau=1$ surface, $R_{1}$, for the winds in our standard model. A distance of $1 \mathrm{kpc}$ is assumed, and the winds are assumed to be smooth.

\begin{tabular}{llll}
\hline \hline Parameter & $v(\mathrm{GHz})$ & $\mathrm{WR}$ & $\mathrm{O}$ \\
\hline$S_{\mathrm{ff}}(\mathrm{mJy})$ & 1.6 & 0.322 & 0.072 \\
& 5.0 & 0.637 & 0.143 \\
& 15.0 & 1.216 & 0.274 \\
$R_{1}\left(10^{14} \mathrm{~cm}\right)$ & 1.6 & 4.07 & 1.93 \\
& 5.0 & 1.83 & 0.87 \\
& 15.0 & 0.84 & 0.40 \\
\hline
\end{tabular}

discussed by Wright \& Barlow (1975), the thermal radio flux for a radially symmetric, isothermal wind moving at constant velocity is given by

$S_{\mathrm{ff}}=23.2\left(\frac{\dot{M}}{\mu_{\mathrm{i}} v_{\infty}}\right)^{4 / 3} \gamma_{\mathrm{e}}^{2 / 3} g_{\mathrm{ff}}^{2 / 3} Z_{\mathrm{i}}^{4 / 3} \frac{v^{2 / 3}}{D^{2}} \mathrm{mJy}$,

where $\dot{M}$ is in units of $M_{\odot} \mathrm{yr}^{-1}, v_{\infty}$ is in $\mathrm{km} \mathrm{s}^{-1}$, the distance to the system, $D$, is in kpc, and $v$ is in $\mathrm{Hz} . Z_{\mathrm{i}}$ is the rms charge of the ions, $\gamma_{\mathrm{e}}$ is the ratio of electron to ion number density (i.e. $\left.n_{\mathrm{e}}=\gamma_{\mathrm{e}} n_{\mathrm{i}}\right), \mu_{\mathrm{i}}$ is the mean mass per ion (in a.m.u.), and $g_{\mathrm{ff}}$ is the free-free Gaunt factor. The radius at which a given optical depth from infinity, $\tau$, occurs, is given by

$R_{\tau}=1.75 \times 10^{28} \frac{\gamma_{\mathrm{e}}^{1 / 3} g_{\mathrm{ff}}^{1 / 3} Z_{\mathrm{i}}^{2 / 3}}{T^{1 / 2} \tau^{1 / 3}}\left(\frac{\dot{M}}{\mu_{\mathrm{i}} v_{\infty} v}\right)^{2 / 3} \mathrm{~cm}$,

where $T$ is in K. $R_{0.242}$ is equivalent to the "characteristic radius" defined by Wright \& Barlow (1975).

If the wind is clumped (see also discussion in Sect. 2.4) the thermal emission from the star mimics an increased mass-loss rate, $\dot{M}=\dot{M}_{\text {actual }} / \sqrt{f}$, where $f$ is the volume filling factor of the clumps (Lamers \& Waters 1984). Values of the rms charge of the ions $\left(Z_{\mathrm{i}}\right)$, the ratio of electron to ion number density $\left(\gamma_{\mathrm{e}}\right)$, and the mean mass per ion $\left(\mu_{\mathrm{i}}\right)$ for the standard model are 1.12, 1.09 and 4.88 for the WR star, and 1.00, 1.00 and 1.29 for the O star. Values of $S_{\mathrm{ff}}$, and $R_{\tau=1}=R_{1}$ are given in Table 1 .

\subsection{The salient features of the standard model}

Figures 2 and 3 illustrate the salient features of our modelling. The density distribution of the unshocked stellar winds and WCR in our standard model is shown in Fig. 2. Two locations in the post-shock flow are highlighted, one near the stagnation point ("A"), and another ("B") at an off-axis position. Comparison of Figs. $3 \mathrm{a}$ and $3 \mathrm{~b}$ yields several points worth noting. First, because the non-thermal electron energy density at "A" is greater than that at "B", the "initial" energy spectrum (dotted line) has a higher normalization in Fig. 3a. Second, the effects of IC and ionic cooling are more significant at "A" (solid lines). IC cooling is high for flow close to the stars since the intensity of the radiation field is high, and near the stagnation point the flow speed is also low, so the exposure time is longer. In the standard model, IC cooling is responsible for the decline in $\gamma_{\max }$ from $10^{5}$ (the assumed initial value) to approximately 200 for flow near the stagnation point (Fig. 3a), while for the off-axis position this decline stops at $\approx 4000$ (Fig. 3b). 


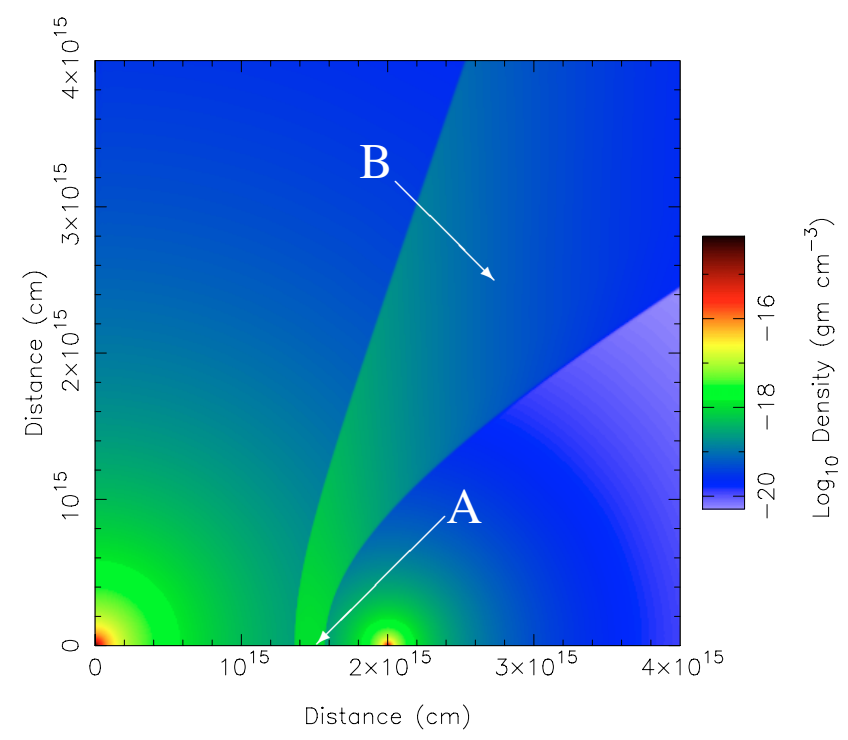

Fig. 2. A density plot of the standard model (showing only the positive $z$-region). The WR star is at $(0,0)$. To aid subsequent discussion, two points in the post-shock flow are highlighted: a position close to the stagnation point (position "A"), and a position some distance off-axis in the shocked gas (position "B").

The turn down in the electron spectrum at low $\gamma$ seen in Figs. 3a and $3 b$ is due to ionic cooling. This effect is again strongest for flow near the stagnation point due to the higher ionic density and slower flow speeds which exist there.

The magneto-bremsstrahlung emission resulting from the "cooled" relativistic energy spectra in Figs. 3a and $3 b$ are shown by the solid lines in Figs. $3 c$ and $3 d$ respectively. The higher energy electrons that reside at position " $\mathrm{B}$ " produce magneto-bremsstrahlung emission extending to higher frequencies $\left(\sim 10^{3} \mathrm{GHz}\right.$, versus $\sim 10 \mathrm{GHz}$ at position "A"; see also Eqs. (9) and (10)). Thus, flow positions subject to high IC cooling produce magneto-bremsstrahlung emission over a smaller range in frequency than positions with less cooling. With the Razin effect included, a low-frequency cut-off to the magneto-bremsstrahlung spectra is seen as a result of its debeaming influence, as shown by the dashed curves in Figs. $3 \mathrm{c}$ and $3 \mathrm{~d}$. It is also worth bearing in mind that the lower normalization of the relativistic energy spectrum at "B" (Fig. 3b) versus "A" (Fig. 3a) means that at $1 \mathrm{GHz}$ the emissivity per unit volume is over an order of magnitude higher at position " $\mathrm{A}$ ".

\subsection{Spectral and intensity distributions}

The spectra and intensity distributions obtained from our standard model are shown in Fig. 4 and the left column of Fig. 5, respectively. For simplicity, the only absorption process included in Figs. 4 and 5 is free-free absorption. Synchrotron emission dominates the total flux below $1 \mathrm{GHz}$. The turnover seen near $400 \mathrm{MHz}$ is due to free-free absorption from the unshocked stellar winds. Above $400 \mathrm{MHz}$, the synchrotron spectrum is a power-law with a spectral index ${ }^{10} \alpha=-(p-1) / 2=-0.5$ for $p=2$ (see Sect. 2). The thermal spectrum is also a power-law

\footnotetext{
10 The flux $S_{v}$ at frequency $v, S_{v} \propto v^{\alpha}$, where $\alpha$ is the spectral index.
}

with a spectral index of +0.6 , as expected. The slight departure of the thermal emission from a power-law at low frequencies is due to the finite size of our hydrodynamical grid. The thermal flux is essentially the sum of the theoretical flux from each wind (cf. Table 1). However, this is not always the case (see Sect. 3.7 and Figs. 10 and 11).

The effect of IC cooling on spectra from our standard model is shown by the dot-dash line in Fig. 4. Its effect is greatest at high frequencies, as demonstrated by the marked decrease of the synchrotron spectral component. However, while this effect is most severe at high frequencies, IC cooling reduces the flux in the entire $0.1-100 \mathrm{GHz}$ frequency range. It is interesting that we do not see a break in the synchrotron spectrum, in spite of its clear signature in Figs. $3 \mathrm{c}$ and $3 \mathrm{~d}$. This is because the frequency of the break in the synchrotron spectrum is spatially dependent (compare Figs. 3c and 3d), and once integrated over the entire WCR the frequency break is smeared out and thus not visible in the final spectrum. A clearly defined break frequency is also lacking in single star models where the synchrotron emission arises from shocks intrinsic to the wind (Van Loo et al. 2005).

Spatially, the effect of IC cooling is to reduce the brightness of the synchrotron emission from the WCR, but more so near the CD than near the shocks (see Fig. 5).

\subsection{Impact of IC cooling and binary separation on the synchrotron emission}

The dramatic effect of IC cooling on the resulting spectra and intensity distributions should be even more pronounced when the separation between the stars is smaller. In Fig. 6 we show the increasing effect of IC cooling on the synchrotron emission as the stellar separation is decreased from $D_{\text {sep }}=2 \times 10^{15} \mathrm{~cm}$ to $D_{\text {sep }}=2 \times 10^{14} \mathrm{~cm}$. Since the intrinsic synchrotron flux scales as $D_{\text {sep }}^{-1 / 2}$, we have normalized the pre-IC cooled spectrum of each model to that obtained at $D_{\text {sep }}=2 \times 10^{15} \mathrm{~cm}$. This allows easier comparison of the divergence between the intrinsic and IC cooled spectra as a function of $D_{\text {sep }}$. The clear trend is for the IC cooled synchrotron spectra to steepen with decreasing $D_{\text {sep }}$.

The spectra and intensity distributions from our full model (including IC cooling, the Razin effect, SSA and free-free absorption) as a function of stellar separation are shown in Figs. 7 and 8 . While the intrinsic synchrotron emission increases with decreasing $D_{\text {sep }}$, the influence of IC cooling (at high frequencies) and free-free absorption and the Razin effect (at low frequencies) means that the observed synchrotron emission may decrease with decreasing separation, which is consistent with the non-detection of non-thermal emission from short period CWB systems.

The low-frequency turnover in Fig. 7 is completely due to the Razin effect. Since $n_{\mathrm{e}} \propto \dot{M} D_{\text {sep }}^{-2}$, and $B \propto \zeta^{1 / 2} \dot{M}^{1 / 2} D_{\text {sep }}^{-1}$, then $v_{\mathrm{R}} \propto \zeta^{-1 / 2} \dot{M}^{1 / 2} D_{\text {sep }}^{-1}$, and we expect the turnover to move to higher frequencies as the separation decreases. This is indeed observed, and the turnover frequencies are in excellent agreement with Eq. (14). In contrast, the turnover frequency due to SSA (cf. Eq. (12)) scales as $v_{\mathrm{SSA}} \propto \zeta^{2 / 3} \dot{M}^{2 / 3} D_{\text {sep }}^{-1}$, since $S_{v} \propto \zeta^{7 / 4} \dot{M}^{7 / 4} D_{\text {sep }}^{-1 / 2}$ and $\Omega \propto D_{\text {sep }}^{2}$. Therefore the turnover 

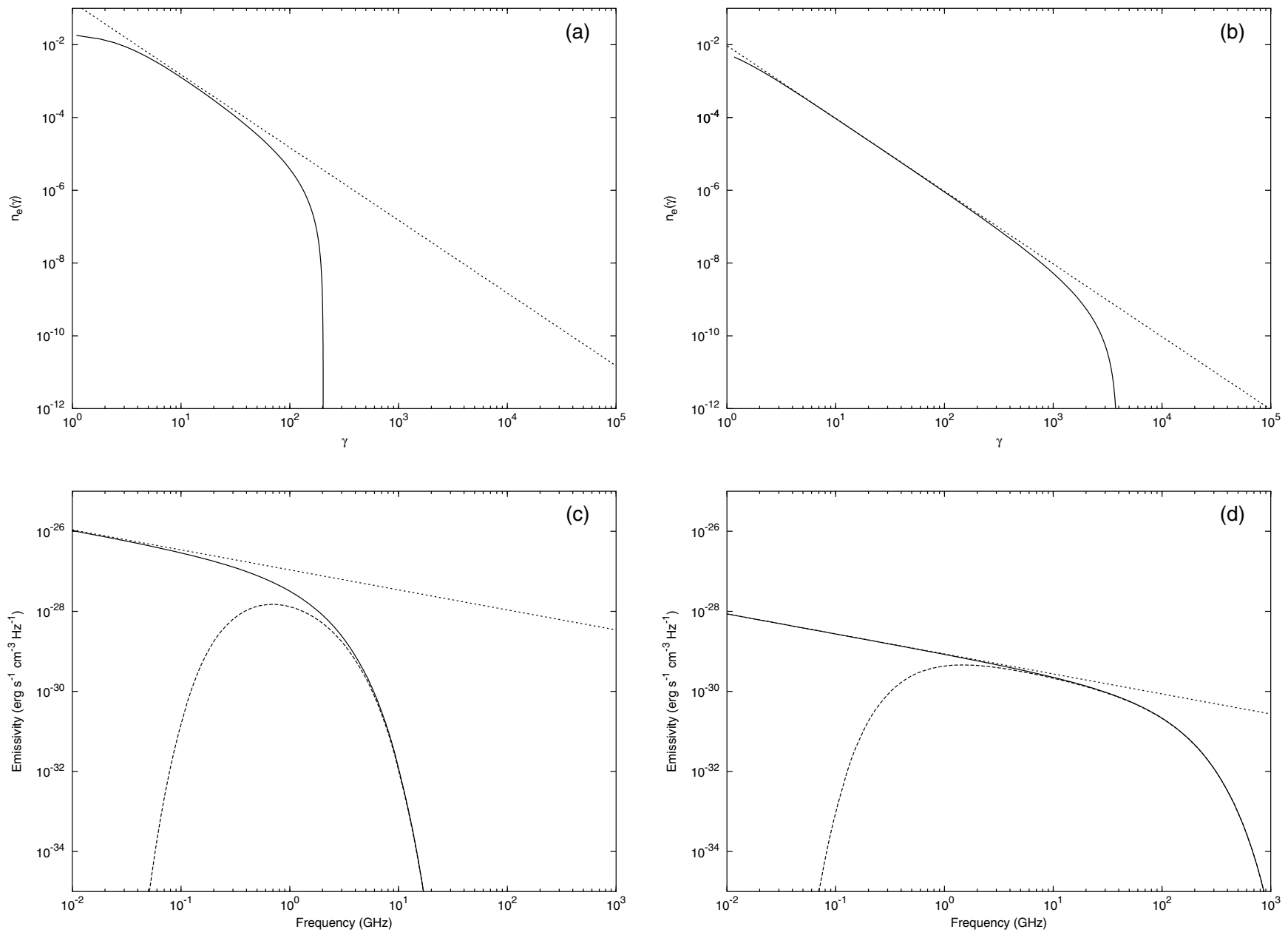

Fig. 3. The effect of IC and ionic cooling on the relativistic electron energy spectrum (panels a) and b)) and on the resulting synchrotron emission (panels c) and d)) for a position close to the stagnation point (position "A", left panels) and some distance off-axis in the shocked gas (position "B", right panels), as indicated in Fig. 2. In a) and b) the dotted line shows the $p=2$ power-law spectrum assuming no cooling, while the solid line shows the effects of IC (high energy cut-off) and ionic cooling (low energy roll-off). In c) and d) the magneto-bremsstrahlung emissivity resulting from these energy distributions is shown. The low frequency cutoff can be influenced by a number of processes. Here, the dashed curves show the Razin effect as an example. The emissivity when IC and ionic cooling are or are not included is shown as a solid and dotted curve respectively. The difference between the solid and dotted curves indicates the improvements in this paper relative to Paper I.

frequencies resulting from the Razin effect and SSA are both $\propto 1 / D_{\text {sep }}$. If we ignore free-free absorption for the moment, we find that the low-frequency turnover in our standard model is caused by the Razin effect rather than SSA when $\zeta_{B}=\zeta_{\text {rel }} \lesssim$ $10^{-2}$ (departures from equipartition are examined in the next subsection). Since $v_{\mathrm{SSA}} / v_{\mathrm{R}} \propto \zeta^{7 / 6} \dot{M}^{1 / 6}$, the SSA turnover frequency may exceed $\nu_{\mathrm{R}}$ when $\dot{M}$ and particularly $\zeta$ are large. The turnover frequency due to free-free absorption depends on the viewing angle into the system, and as it has a steeper dependence on $D_{\text {sep }}$ than either the Razin effect or SSA $\left(v_{\mathrm{ff}} \propto D_{\text {sep }}^{-10 / 7}\right.$ - see Paper I), it is increasingly likely that the low-frequency turnover will be caused by free-free absorption as $D_{\text {sep }}$ decreases.

At the high frequency end of the spectra in Fig. 7, the difference between the observed and intrinsic synchrotron flux is entirely due to IC cooling. With the $B$-field used in the standard model, the emission at a given frequency arises from electrons with fairly high values of $\gamma$ (cf. Eq. (10)). Thus the influence of
IC cooling is fairly high, and this is reflected in the curvature in the synchrotron spectra at high frequencies. As $D_{\text {sep }}$ decreases, IC cooling becomes increasingly important, and we find that high frequency synchrotron emission is confined to very thin regions immediately behind the shocks. The synchrotron emission behind the WR shock can be appreciably higher than that behind the $\mathrm{O}$ shock, since the non-thermal electrons are further removed from the $\mathrm{O}$ star in this case.

Figure 8 shows that the maximum intensity of the emission from the WCR may increase with decreasing binary separation. However, the bottom panel of Fig. 7 reveals that the synchrotron emission at $1.6 \mathrm{GHz}$ when $D_{\text {sep }}=2 \times 10^{14} \mathrm{~cm}$ is negligible, as it is greatly reduced by the Razin effect. The observed emission from the WCR is therefore dominated by free-free emission (the increase in the free-free emission from the WCR with decreasing separation is investigated further in Sect. 3.7). The fact that the WCR emission at $1.6 \mathrm{GHz}$ changes from synchrotron dominated when $D_{\text {sep }}=2 \times 10^{15} \mathrm{~cm}$, to 


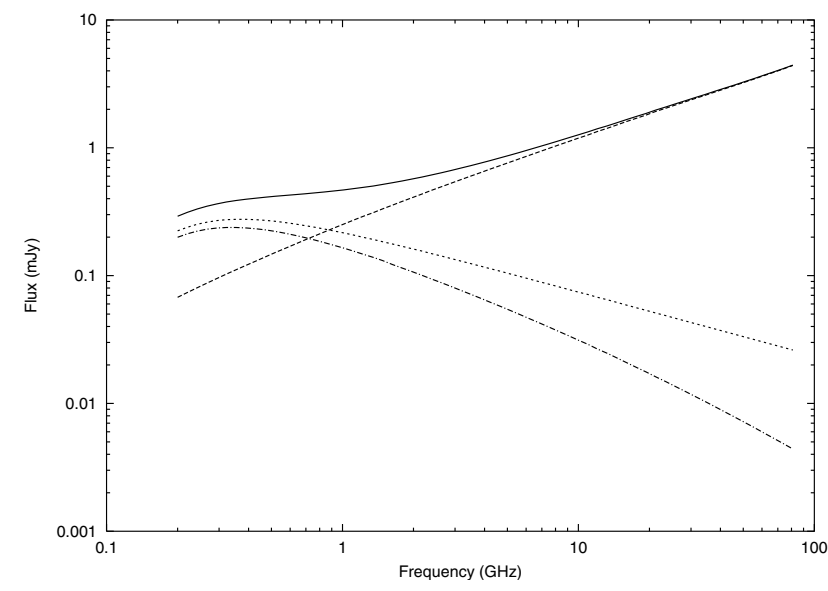

Fig. 4. Spectra from our standard model with a $0^{\circ}$ viewing angle free-free flux (dashed), synchrotron flux (dotted), and total flux (solid). We do not include IC cooling, the Razin effect or SSA. With IC cooling included (dot-dash) a clear reduction in the amount of synchrotron emission is seen.

free-free dominated when $D_{\text {sep }}=2 \times 10^{14} \mathrm{~cm}$, also explains why the "V"-shaped emission from the WCR seen at $D_{\text {sep }}=$ $2 \times 10^{15} \mathrm{~cm}$ disappears at $D_{\text {sep }}=2 \times 10^{14} \mathrm{~cm}$ (see Fig. 8).

Figure 8 also shows that the emission from the WCR is sometimes brightest to either side of the apex of the WCR, due to high absorption. This is sometimes seen in observations (e.g., Watson et al. 2002; Dougherty et al. 2005), though it may also arise from time variability in the structure of the WCR.

\subsection{Departures from equipartition}

So far, we have assumed that the energy density of the nonthermal electrons and the $B$-field are equal. This assumption was made for the sake of convenience, but it is quite probable that this condition is not attained. For example, the $B$-field energy density may be equal to the total non-thermal energy density from electrons and ions. In fact, some astrophysical sources are far from having $U_{\text {rel }}=U_{B}$ (e.g., in some very compact radio sources, Kellermann \& Pauliny-Toth (1981) note that $U_{\text {rel }} / U_{B}>10^{10}$ !). We have therefore explored the effect of varying the ratio $\zeta_{B} / \zeta_{\text {rel }}$. In essence, this allows us to explore how changes in the $B$-field affect the emission. The energy density of non-thermal ions has no effect on the radio emission.

When $\zeta_{B}$ is kept constant and $\zeta_{\text {rel }}$ varied, the synchrotron spectrum simply scales in flux as $S_{v} \propto \zeta_{\text {rel }}$ (see Eq. (6) in Paper I). However, when $\zeta_{\text {rel }}$ is kept constant and $\zeta_{B}$ varied the turnover frequency changes as well as the normalization, since $v_{\mathrm{R}} \propto 1 / B \propto 1 / \sqrt{\zeta_{B}}$. At high values of $\zeta_{B}$ we expect SSA to succeed the Razin effect as the dominant absorption process behind the low frequency turnover in the synchrotron spectrum. When this happens we expect the turnover frequency to scale as $v_{\mathrm{SSA}} \propto B^{1 / 6}$ (see Eq. (12)). Our calculations show that it is relatively easy to achieve a turnover in the synchrotron spectrum at fairly high frequencies through the Razin effect by reducing $\zeta_{B}$, but difficult to achieve turnovers through SSA even a little above that obtained when $\zeta_{B}=\zeta_{\text {rel }}$ since the dependence of $v_{\mathrm{SSA}}$ on $B$ is weak, and requires commensurately large values of $B$. This is illustrated in Fig. 9 where we plot the normalized turnover frequency as a function of $\zeta_{B} / \zeta_{\text {rel }}$.

\subsection{The thermal flux and binary separation}

The effect of binary separation on the thermal flux from the WCR has not previously been considered. As noted by Stevens (1995), the free-free emissivity from the WCR is slightly enhanced compared to that from the unshocked winds, at an equivalent distance from the system centre, since $\varepsilon_{v}^{\mathrm{ff}} \propto$ $\rho^{2} T^{-1 / 2} g_{\mathrm{ff}}$. In the WCR the density is typically $4 \times$ higher (cf. the jump conditions for a strong shock), the temperature is of order $10^{2}-10^{4} \times$ higher, with perhaps a typical increase of a factor of $10^{3}$, and the Gaunt factor is approximately double that of the ambient gas. In contrast, the free-free absorption is reduced by a factor of $\sim 10^{3}$, since $\alpha_{v}^{\mathrm{ff}} \propto \rho^{2} T^{-3 / 2} g_{\mathrm{ff}}$. If the unshocked winds are clumpy, and the gas in the WCR basically smooth (see Sect. 2.4), the difference in absorption can be even larger.

In Fig. 10 we show the relative contribution to the total thermal emission of the unshocked winds and the WCR. Notably, the high frequency thermal spectrum of the WCR has a spectral index which is indicative of optically thin thermal emission, quite unlike that from the unshocked stellar wind. The intrinsic thermal emission from the WCR is strongly absorbed at low frequencies, mostly by free-free absorption from the unshocked winds (i.e. from gas external to the WCR), with a small contribution from absorption internal to the WCR. The slight curvature in the unshocked wind emission at low frequencies is a result of the finite size of the computational grid.

We find that the thermal emission from the WCR increases as the binary separation decreases, while the thermal emission from the unshocked winds remains broadly the same. Since $\varepsilon_{v}^{\mathrm{ff}} \propto \rho^{2} \propto D_{\text {sep }}^{-4}$ and the volume of the WCR scales as $D_{\text {sep }}^{3}$, the thermal emission from the WCR scales as $D_{\text {sep }}^{-1}$. Since the emission from the WCR is optically thin, we see a substantial fraction of this emission at certain viewing angles, and at small separations the contribution of the WCR to the total thermal flux can be significant. A dramatic consequence of this is that a composite-like spectrum (Fig. 10), often presumed to be evidence for non-thermal emission, can result entirely from thermal processes! The spectral index between 5 and $15 \mathrm{GHz}$ of the total emission in Fig. 10 is $\approx 0.4$, with the result that the freefree emission from this model would be classified as a "composite" spectrum if it was included in the observational analysis of Dougherty \& Williams (2000). Nevertheless, we believe that a non-thermal origin is the most likely explanation in all of the systems noted in Dougherty \& Williams (2000) since these are typically wide systems.

In Fig. 11a we show how the total thermal flux varies with binary separation and viewing angle at $v=15 \mathrm{GHz}$. At wide separations and a $0^{\circ}$ viewing angle, the total thermal flux from the system (the sum of the thermal emission from the unshocked winds and from the WCR) approaches the sum of the theoretical free-free flux from each stellar wind. This is because the WCR occurs well outside the $R_{1}$ radius of emission of each wind (see Table 1), and its contribution to the emission is negligible since the intrinsic thermal emission from the WCR scales 

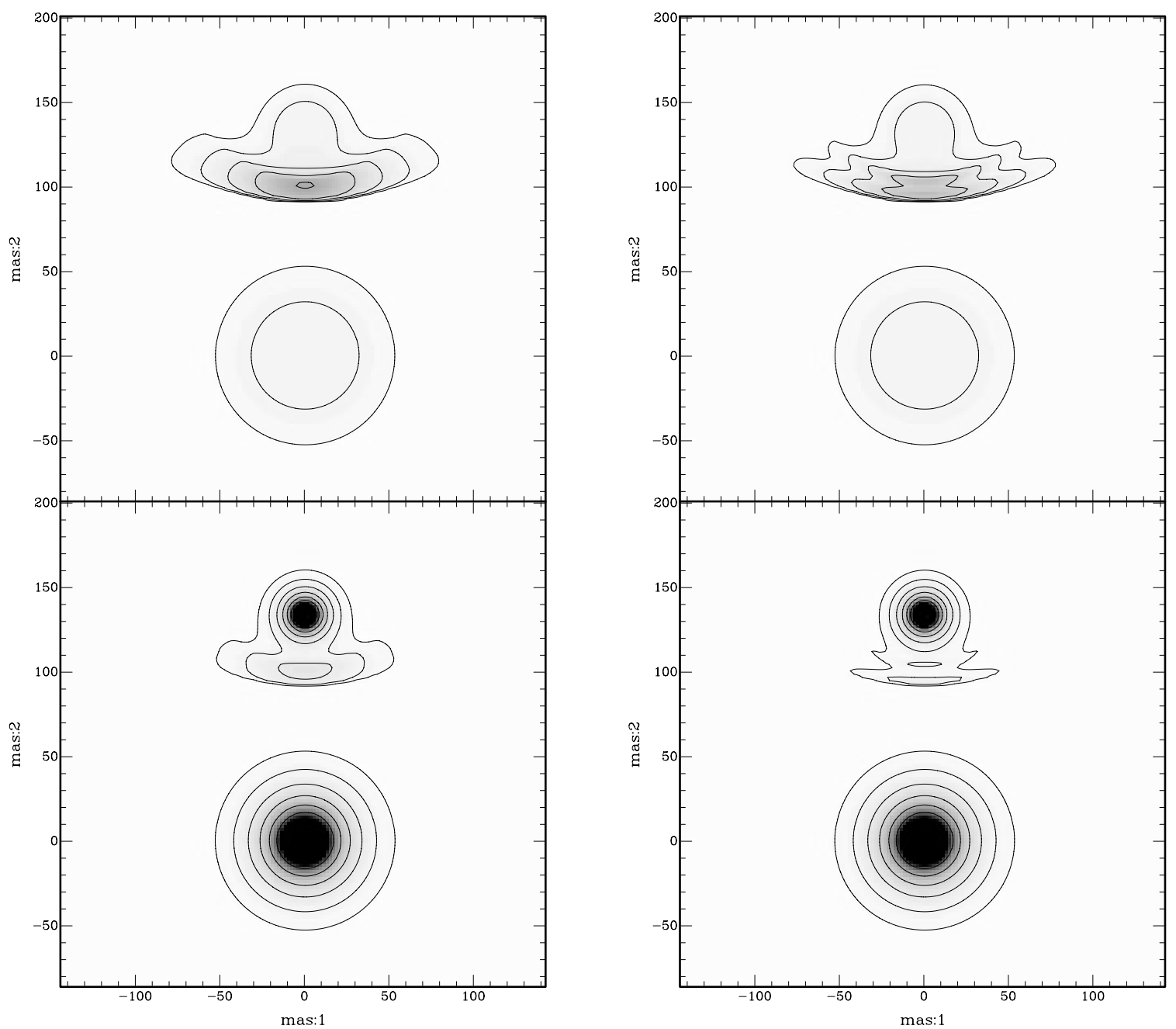

Fig. 5. The impact of IC cooling on the intensity distributions of our standard model, for a viewing angle of $0^{\circ}$ and at $1.6 \mathrm{GHz}(t o p)$ and $22 \mathrm{GHz}$ (bottom). Neither the Razin effect nor SSA are included in this calculation. The images on the left do not include IC cooling, while those on the right do. Each image has the same intensity scale and contours.

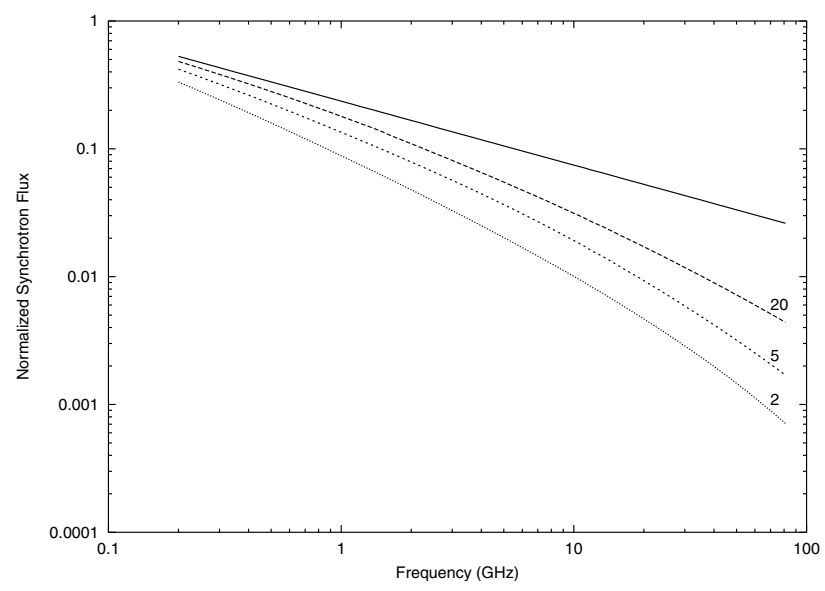

Fig. 6. The effect of separation on the IC cooled synchrotron spectra with the standard model, a $0^{\circ}$ viewing angle and $\zeta=10^{-4}$, for $D_{\text {sep }}=2,5$, and $20 \times 10^{14} \mathrm{~cm}$. The spectra are normalized so that the intrinsic emission spectra (i.e. without free-free absorption, SSA, the Razin effect, or IC cooling) are equal to the intrinsic spectrum at $D_{\text {sep }}=20 \times 10^{14} \mathrm{~cm}$ (solid line).

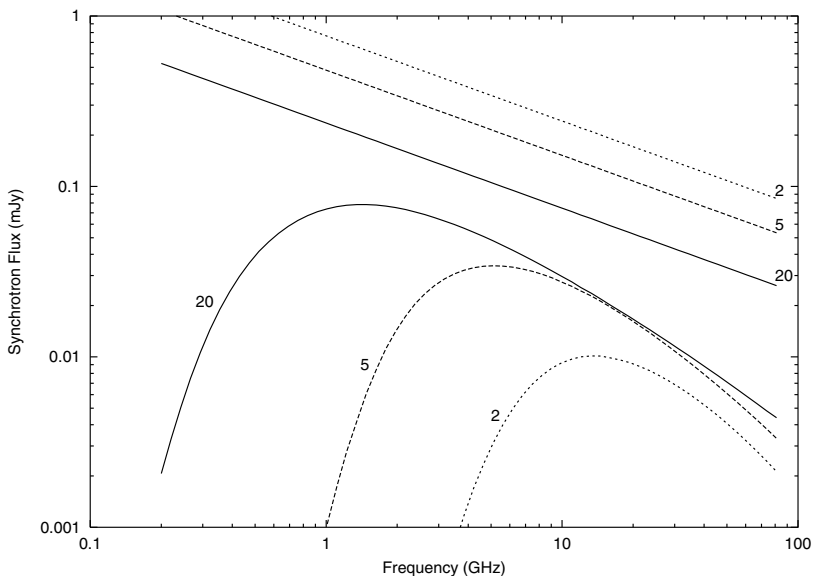

Fig. 7. The effect of separation on the synchrotron spectra with the standard model, a $0^{\circ}$ viewing angle and $\zeta=10^{-4}$, for $D_{\text {sep }}=2,5$, and $20 \times 10^{14} \mathrm{~cm}$. In each case the pre-IC cooled intrinsic synchrotron spectrum and the observed IC-cooled synchrotron spectrum are shown. 


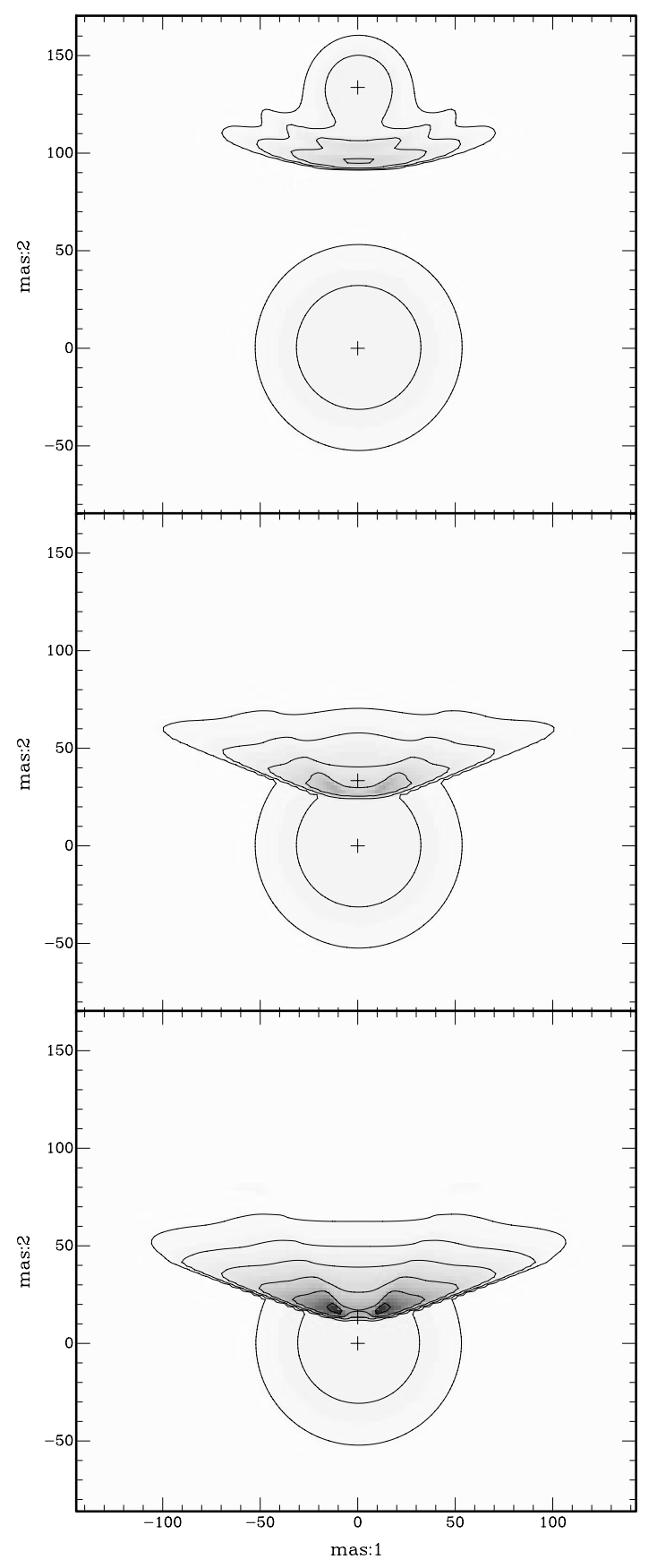

Fig. 8. Intensity distributions at $1.6 \mathrm{GHz}$ and a $0^{\circ}$ viewing angle with a binary separation of 20 (top), 5 (middle), and $2 \times 10^{14} \mathrm{~cm}$ (bottom), corresponding to angular separations of 133,33 and 13.3 mas respectively. The crosses denote the positions of the stars, with the WR star located at $(0,0)$. Each image has the same intensity scale and contours. IC cooling, the Razin effect, SSA, and free-free absorption are included, and $\zeta=10^{-4}$. Absorption by the WR wind is highest at the apex of the WCR - as the binary separation decreases, the observed intensity from the WCR reaches maxima either side of the symmetry axis. The nature of the emission from the WCR also changes with binary separation. In the top panel it is predominantly non-thermal, while in the bottom panel it is completely thermal. This dramatic change is due to the Razin turnover frequency scaling as $\nu_{\mathrm{R}} \propto D_{\text {sep }}^{-1}$.

as $D_{\text {sep. }}^{-1}$. In closer systems, the WCR moves within the $R_{1}$ radius of each wind and removes a segment of their emission.

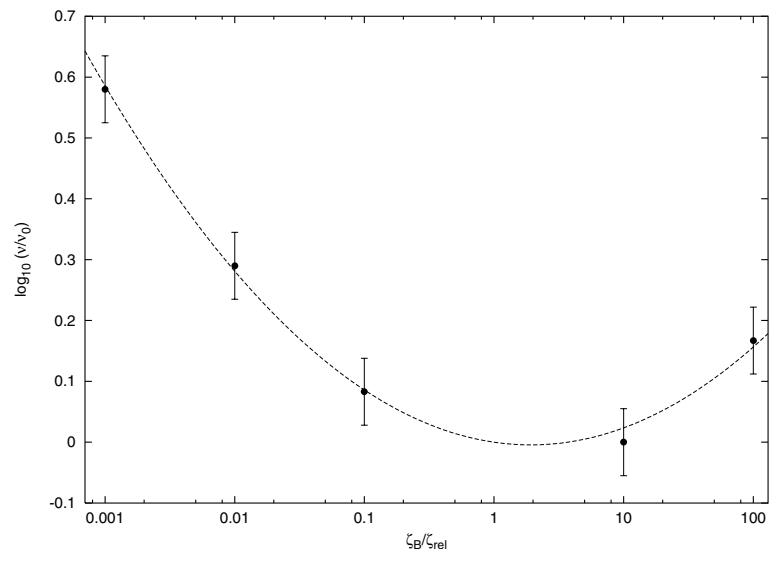

Fig. 9. The synchrotron turnover frequency (normalized to the turnover frequency when $\left.\zeta_{B}=\zeta_{\text {rel }}\right)$ as a function of $\zeta_{B} / \zeta_{\text {rel }}$. Note that $\zeta_{\text {rel }}$ was kept constant in these calculations. We estimate the uncertainty of each data point based on the finite frequency sampling in our calculations. The curve is a least squares fit of a second order polynomial in $\log _{10}\left(\zeta_{B} / \zeta_{\text {rel }}\right)$ to $\log _{10}\left(v / v_{0}\right)$, and is forced to go through the position $(0.0,0.0)$. The coefficient's are $0.055,-0.031$ and 0.0 .

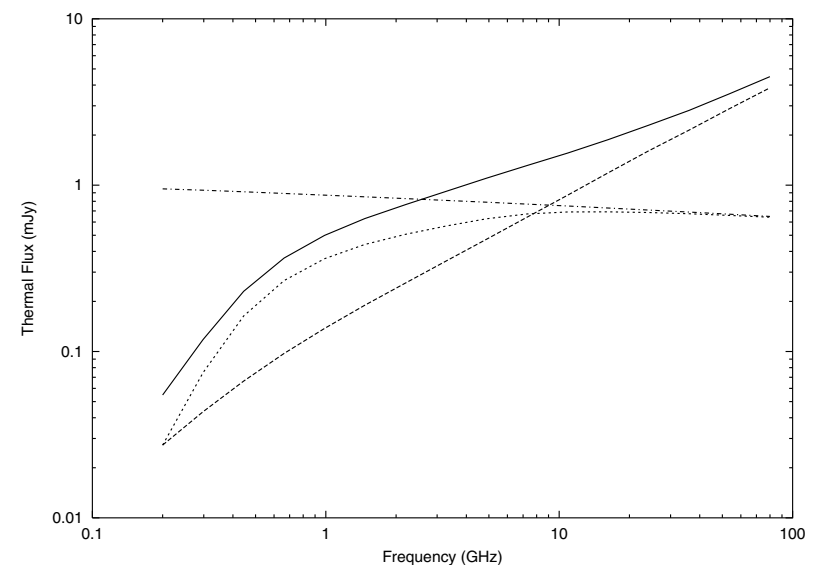

Fig. 10. The thermal emission from the standard model with a $0^{\circ}$ viewing angle and a binary separation of $2 \times 10^{14} \mathrm{~cm}$. The thermal emission from the unshocked winds (dashed), the WCR (dotted), and the total thermal emission (solid) are shown. The intrinsic thermal emission from the WCR (before free-free absorption) is shown by the dotdashed line. Synchrotron emission is not displayed.

However, the resulting reduction in flux from the unshocked winds is more than compensated for by the increase in thermal emission from the WCR. Therefore, the total thermal emission can considerably exceed that expected from the two stellar winds. For example, when $D_{\text {sep }}=2 \times 10^{14} \mathrm{~cm}$, the thermal flux from the standard model exceeds the total theoretical thermal flux by more than $20 \%$. The excess could be considerably higher at smaller separations and lower frequencies. The effect is smaller at higher frequencies, since $R_{1} \propto v^{-0.7}$. Figure 11a also shows that the observed flux is reduced when one star is in front of the other $\left( \pm 90^{\circ}\right)$, as one would expect.

The nature and degree of clumping may also affect how the free-free flux scales with binary separation. If the unshocked winds are clumpy, but the WCR smooth due to rapid destruction of the clumps, the thermal flux may actually decrease with 

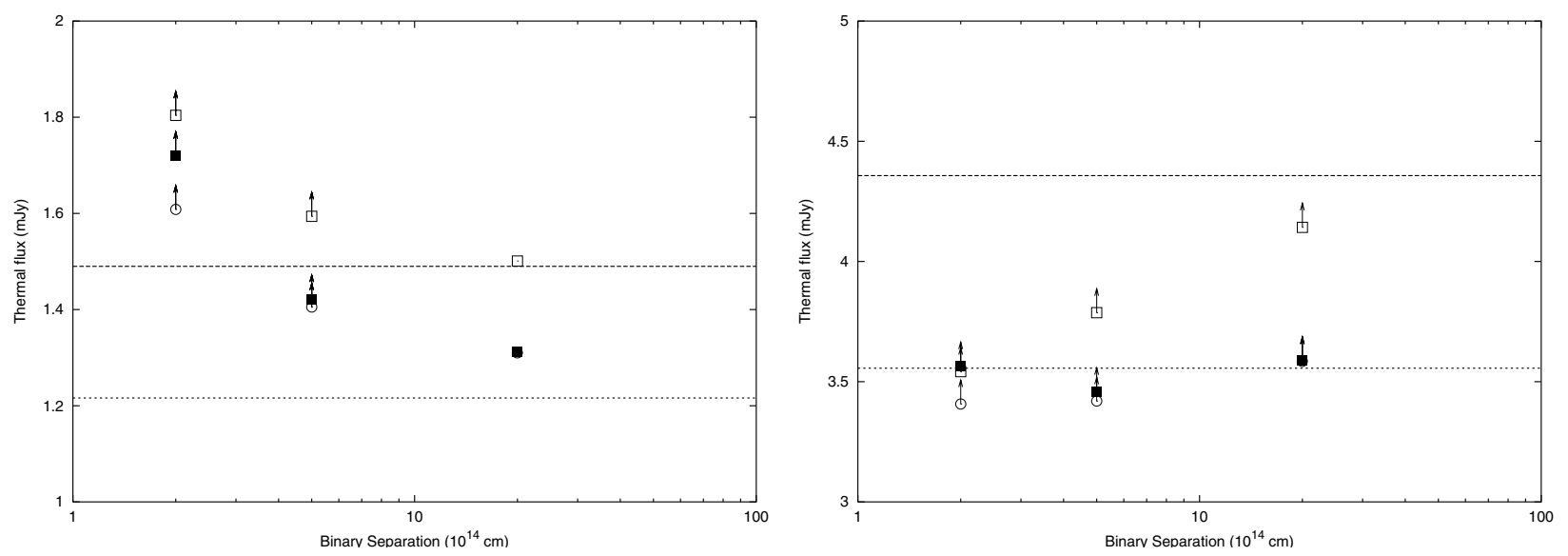

Fig. 11. a) The thermal flux from our standard model at $15 \mathrm{GHz}$ and various viewing angles as a function of the binary separation. The dotted line shows the theoretical flux from the WR star, while the dashed line shows the theoretical flux from the WR and O star combined. The open circles are for a viewing angle of $-90^{\circ}$ (WR star in front), the open squares are for $0^{\circ}$, and the filled squares are for $90^{\circ}(\mathrm{O}$ star in front). The points at $2 \times 10^{14} \mathrm{~cm}$ and $5 \times 10^{14} \mathrm{~cm}$ are plotted as lower limits, since not all of the emission from the WCR is captured in the calculations. b) As a), but for clumpy winds with a filling factor of 0.2. In both panels the open circle at $2 \times 10^{15} \mathrm{~cm}$ is hidden underneath the filled square.
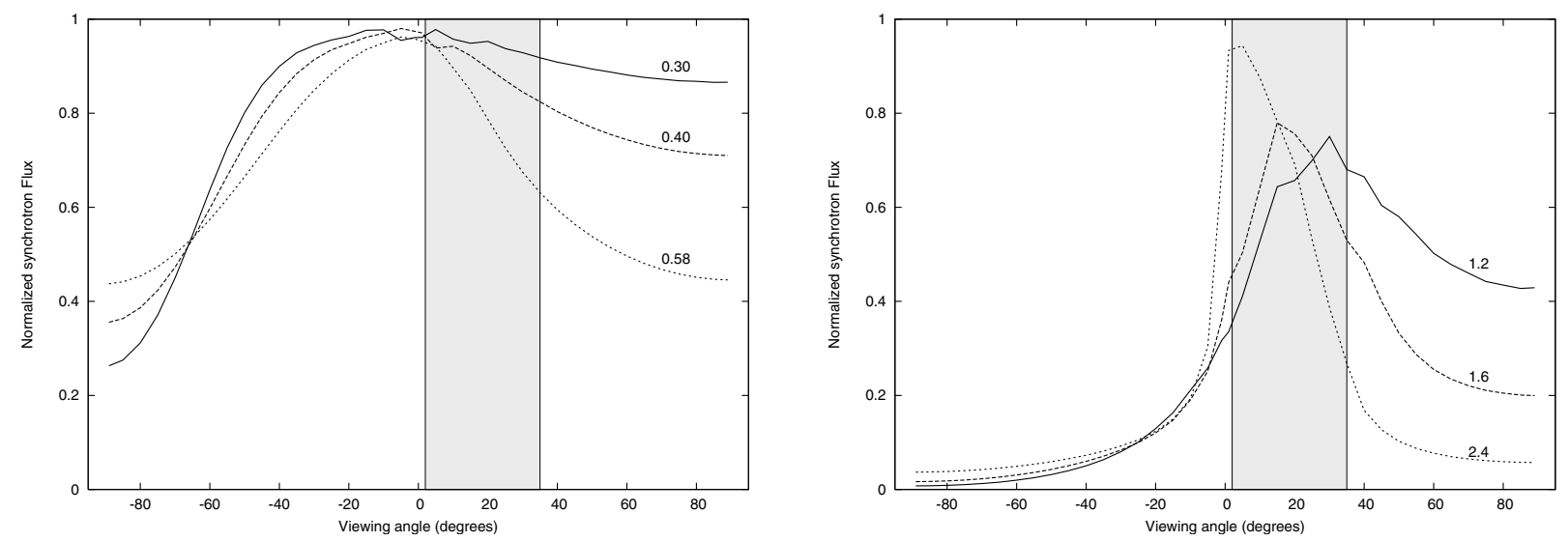

Fig. 12. a) The change in the synchrotron flux at $1.6 \mathrm{GHz}$ as a function of viewing angle using the standard model parameters with $\zeta=10^{-4}$, but where $\dot{M}_{\mathrm{O}}$ is adjusted to vary $\eta$. Shown are curves for $\eta=0.0316$ (solid), $\eta=0.1$ (dashed), and $\eta=0.316$ (dotted). Each curve is normalized to the intrinsic synchrotron emission in each model. SSA, the Razin effect, and IC cooling are included. The binary separation is $2 \times 10^{15} \mathrm{~cm}$. At a viewing angle of $-90^{\circ}$, the WR star is in front; at $0^{\circ}$ the lines of sight are perpendicular to the axis of symmetry of the model; and at $+90^{\circ}$ the $\mathrm{O}$ star is in front. Values for $R_{1}^{\mathrm{O}} / r_{\mathrm{O}}$ are noted next to each curve. The angular range of the WCR for the model with $\eta=0.316$ is shown by the shaded region, with the asymptotic angles of the WR and $\mathrm{O}$ shocks delimiting its edges. b) As a) but where the binary separation (hence $r_{\mathrm{O}}$ ) is reduced by a factor of 4 to $5 \times 10^{14} \mathrm{~cm}$.

decreasing separation, as shown in Fig. 11b. This is because as the stars move closer together, the increased emission from the WCR is more than offset by the decrease in the overall amount of clumpy material in the system. Whether the thermal flux increases with decreasing binary separation in a particular system depends on the nature and degree of clumping in that system.

\subsection{Synchrotron emission and wind-momentum ratio}

Free-free absorption by the surrounding stellar winds can have a large affect on the observable low frequency synchrotron emission. In previous models where the non-thermal emission is treated as arising from a point source, the free-free absorption by the stellar winds is determined along a single line of sight. Since the spatial extent of the WCR is ignored in such models, the low opacity within the WCR is also disregarded. In our models, the spatial extent of the WCR means that there are multiple lines-of-sight to the WCR. The amount of free-free absorption that the synchrotron emission suffers varies with viewing angle, but is also sensitive to the wind momentum ratio, $\eta$, since it is the combination of these parameters that determines how lines of sight into the system are aligned with the opening angle of the WCR.

In Fig. 12a we show how the synchrotron flux at $1.6 \mathrm{GHz}$ varies with viewing angle and $\eta$ when the binary separation is $2 \times 10^{15} \mathrm{~cm}$. The half-opening angle of the WR shock, the $\mathrm{CD}$, and the $\mathrm{O}$ shock as a function of $\eta$ are noted in Table $2^{11}$.

${ }_{11}$ Eichler \& Usov (1993) provide an analytical expression for the half-opening angle of the $\mathrm{CD}, \theta_{\mathrm{cd}}$ (their Eq. (3)). However, the statement that the angle between the two shocks, $\Delta \theta \sim \theta$, is inaccurate, as the data in Table 2 shows. Instead we find that $\Delta \theta \approx 35-40^{\circ}$ for the range of $\eta$ noted in Table 2, whereas $\theta$ changes by almost a factor of 2 . 
Table 2. Half-opening angles ( $\theta$, in degrees, measured from the line of centres between the stars in our models), of the shocks in the WR and $\mathrm{O}$ winds bounding the WCR, and of the contact discontinuity, as a function of $\eta$.

\begin{tabular}{llll}
\hline \hline$\eta$ & WR & CD & O \\
\hline 0.3160 & 88 & 69 & 55 \\
0.1000 & 74 & 52 & 36 \\
0.0316 & 56 & 36 & 17 \\
\hline
\end{tabular}

The main characteristic is an increase in absorption of the synchrotron emission when the lines of sight to the apex of the WCR pass close to either star.

Here we vary $\eta$ by changing $\dot{M}_{\mathrm{O}}$, so it is not surprising to see that the absorption at $+90^{\circ}$ is highest when $\eta$ is highest (i.e. $\dot{M}_{\mathrm{O}}$ highest). When the O star is in front, the amount of free-free absorption depends on the ratio of $R_{1}^{\mathrm{O}} / r_{\mathrm{O}}$ (Eichler \& Usov 1993). At $1.6 \mathrm{GHz}$, this ratio is $0.58,0.40$, and 0.30 for $\eta=0.316,0.1$, and 0.0316 , respectively. Larger values of $R_{1}^{\mathrm{O}} / r_{\mathrm{O}}$ result in higher absorption, which is in agreement with Fig. 12a. We naively expected that the synchrotron emission would be least affected by absorption when the observer looks down the WCR into the system. However, we find that in all 3 cases, the absorption is a minimum at viewing angles which are close to the asymptotic angle of the WR shock, and steadily increases as the viewing angle swings from being parallel to the WR shock to being parallel to the $\mathrm{O}$ shock. We attribute this finding to the fact that even when the viewing angle is within the WCR, the far side of the WCR is absorbed by the O star wind, and note that there are implications for any observing strategy attempting to detect radio bursts in CWB systems (e.g., in $\gamma^{2}$ Velorum).

It is also interesting that the model with $\eta=0.316$ shows the lowest relative absorption when the viewing angle is $-90^{\circ}$. We attribute this to the wider opening angle of the WCR in the model, and the fact that more of the WCR is visible when $\eta$ is high. Though not shown here, the variation with viewing angle is reduced at higher frequencies, since $R_{1} \propto v^{-0.7}$.

The variation in synchrotron flux with viewing angle is more pronounced at smaller binary separations, as shown in Fig. $12 \mathrm{~b}$, where the values of $R_{1}^{\mathrm{O}} / r_{\mathrm{O}}$ are $4 \times$ larger than previously. In all of the models in Fig. 12b, the drop in emission as the sightlines move out of the WCR is steeper on the WR side.

If an observer's line of sight into the system lies in the orbital plane, two bursts of radio emission will be seen as the WCR sweeps past, as suggested by Eichler \& Usov (1993). We find that this occurs if $R_{1}^{\mathrm{O}} / r_{\mathrm{O}} \gtrsim 0.4$. When $R_{1}^{\mathrm{O}} / r_{\mathrm{O}} \gtrsim 2$, the burst is confined to a range in viewing angle equal to $\Delta \theta$. However, if the inclination of the orbital plane is such that the line of sight intercepts the limb of the WCR, only one such burst per orbit may be seen. In contrast, the behaviour of the system with $\eta=0.0316$ shown in Fig. 12a is better characterized as a single decline in synchrotron emission when the WR star is in front of the $\mathrm{O}$ star, since the emission remains high even when the $\mathrm{O}$ star is directly in front of the WR star.
The complexity demonstrated in Fig. 12 further illustrates the need for models which take into account the spatial extent of the emission and absorption.

\section{The radio spectrum of WR 147}

Having explored how the radio flux is affected by IC cooling, binary separation, the wind momentum ratio, and variations in the $B$-field, we once again turn our attention to the modelling of a specific system. WR 147 is one of the brightest CWB systems, and is also one of the systems where the stellar wind of the WR star and the WCR have been resolved at radio wavelengths (Williams et al. 1997). In addition, WR 147 has one of the most extensively observed radio spectra of any of the CWB systems, with observations from $353 \mathrm{MHz}$ to $43 \mathrm{GHz}$.

In Paper I, we demonstrated a number of fits of our CWB models to the synchrotron spectrum of WR 147. However, those models suffer from two main problems. Firstly, the optically-thick turnover at $\sim 1 \mathrm{GHz}$ was attributed to SSA which we have discovered subsequently was miscalculated (see earlier footnote), and a new fit with the corrected model code is now required. Secondly, the synchrotron spectrum we were attempting to fit in Paper I had been derived from the observed total fluxes and an extrapolated thermal spectrum for the stellar wind emission based on the flux of the WR star stellar wind at several high frequencies (e.g., Setia Gunawan et al. 2001). Though a reasonable approach, the resulting synchrotron spectrum exhibits considerable variation dependent on the adopted thermal model. In an attempt to address this problem, here we aim to fit the observed total radio emission at each of the observed frequencies, and show the contributing synchrotron and thermal spectra from the model.

Observations of WR 147 are available from a number of authors. These are summarised in Table 3. Skinner et al. (1999) used two methods to obtain flux estimates from the same data, and these show considerable scatter. We have taken the liberty of quoting an average of the values from that paper. It appears that WR 147 is variable at some frequencies (Setia Gunawan et al. 2001), so where observations at multiple epochs are available (e.g., Churchwell et al. 1992; Contreras et al. 1996; Contreras \& Rodríguez 1999), we have quoted an average flux. To attain a reasonable estimate of the uncertainty in the flux values, we quote either the variance of the observations (where there are multiple observations), or an estimate of the uncertainty in the absolute flux scale, whichever is largest. For the uncertainty in the flux scale, we adopt $5 \%$ for frequencies $15 \mathrm{GHz}$ and greater, and $2 \%$ for frequencies less than $15 \mathrm{GHz}$ (Perley \& Taylor 2003). We believe that our approach to estimating the observed fluxes from WR 147 is very conservative, but it at least makes an attempt at assessing the relative merits of all observations in the literature, and takes into account the impact of variability.

WR 147 is a very wide system with a projected separation $D_{\text {sep }} \cos i=0.635 \pm 0.020^{\prime \prime}$ (Williams et al. 1997), where $i$ is the angle at which we view the system. At the estimated distance of $\sim 0.65 \mathrm{kpc}$ (Churchwell et al. 1992; Morris et al. 2000) this corresponds to a separation $D_{\text {sep }} \sim 415 / \cos i$ AU. As noted in Paper I, this relationship between $D_{\text {sep }}$ and $i$ 
Table 3. Observations of WR 147 from the literature.

\begin{tabular}{llllll}
\hline \hline $\begin{array}{l}\text { Freq. } \\
(\mathrm{GHz})\end{array}$ & $\begin{array}{l}\text { Skinner }^{a, 1} \\
(\mathrm{mJy})\end{array}$ & $\begin{array}{l}\text { Setia Gunawan }^{b} \\
(\mathrm{mJy})\end{array}$ & $\begin{array}{l}\text { Contreras }^{c} \\
(\mathrm{mJy})\end{array}$ & $\begin{array}{l}\text { Churchwell }^{d} \\
(\mathrm{mJy})\end{array}$ & $\begin{array}{l}\text { Average } \\
(\mathrm{mJy})\end{array}$ \\
\hline 0.353 & - & $16.0 \pm 4.0$ & - & - & $16.0 \pm 4.0$ \\
1.42 & $25.2 \pm 0.4$ & $26.4 \pm 2.0$ & - & $26.1 \pm 0.1^{3}$ & $25.9 \pm 2.0$ \\
4.86 & $37.3 \pm 1.6$ & $35.4 \pm 0.4$ & $38.4 \pm 0.1$ & $37.5 \pm 1.0^{2}$ & $37.2 \pm 1.6$ \\
8.3 & $42.5 \pm 2.2$ & - & $41.0 \pm 3.7^{2}$ & - & $41.8 \pm 3.7$ \\
14.94 & $47.9 \pm 1.7$ & - & $57.4 \pm 0.3$ & $47.4 \pm 3.2^{2}$ & $50.9 \pm 3.2$ \\
22.46 & $54.7 \pm 2.7$ & - & - & $59.1 \pm 1.2^{3}$ & $56.9 \pm 2.8$ \\
43.0 & $82.8 \pm 1.2$ & - & $82.8 \pm 1.1$ & - & $82.8 \pm 4.1$ \\
\hline
\end{tabular}

References: ${ }^{a}$ Skinner et al. (1999); ${ }^{b}$ Setia Gunawan et al. (2001); ${ }^{c}$ Contreras et al. (1996), and Contreras \& Rodríguez (1999) ${ }^{d}$ Churchwell et al. (1992).

Notes:

${ }^{1}$ Skinner et al. (1999) quote two values for flux using two different techniques on the same data. The value given here is the average value, along with the standard deviation. ${ }^{2}$ Error based on the standard deviation of multiple observations. ${ }^{3}$ Error assumed to be the uncertainty in the images.

represents an important constraint for any models of the system. We use the stellar wind parameters from Paper I: $\dot{M}_{\mathrm{WN} 8}=$ $2 \times 10^{-5} M_{\odot} \mathrm{yr}^{-1}, v_{\infty \mathrm{WN} 8}=950 \mathrm{~km} \mathrm{~s}^{-1}, v_{\infty \mathrm{OB}}=1000 \mathrm{~km} \mathrm{~s}^{-1}$ and $\dot{M}_{\mathrm{OB}}=3.8 \times 10^{-7} M_{\odot} \mathrm{yr}^{-1}$, giving a wind momentum ratio $\eta=0.02$ (Pittard et al. 2002). Of these parameters, $\dot{M}_{\mathrm{OB}}$ is the most uncertain. This is partly due to the considerable uncertainty in the companion's spectral type - estimates range from B0.5 (Williams et al. 1997) to O5-7 I-II (Lépine et al. $2001)$. The luminosity of the companion $\left(L_{\mathrm{OB}}\right)$ ranges from $\sim 10^{5}-10^{6} L_{\odot}$, resulting in considerable scope for varying the degree of IC cooling in the models. The composition of the WN8 stellar wind is taken from Morris et al. (2000), giving $X=0.09, Y=0.89$, and $Z=0.016$. The wind temperature for both stellar winds was assumed to be $10 \mathrm{kK}$, and the dominant ionization states were $\mathrm{H}^{+}, \mathrm{He}^{+}$and $\mathrm{CNO}^{2+}$.

Firstly, we wish to repeat the modelling done in Paper I to evaluate the impact of the miscalculated SSA. The resulting models are shown in Fig. 13. As mentioned earlier, SSA has little impact at the frequencies of the observations in these systems, and we have to turn to other mechanisms to generate the optically thick turnover. With $\zeta_{B}=\zeta_{\text {rel }}$, we find that the low frequency turnover is controlled by free-free absorption along the line-of-sight through the circum-binary stellar wind envelope, which is contrary to the conclusion from Paper I. The line-ofsight opacity changes with the viewing angle of the model: positive and negative viewing angles pass first through the $\mathrm{O}$ star wind and the denser WR star wind respectively. As a result, large negative angles are more opaque than large positive values, resulting in lower observed fluxes at low frequencies with negative viewing angles. The quality of the fits in Fig. 13 is largely determined by the model emission at $353 \mathrm{MHz}$, with little variation between models at all other observed frequencies. The $-30^{\circ}$ model matches the $353 \mathrm{MHz}$ data the best, though we have made no attempt to adjust parameters in the model to improve any of the fits. Rather, models $\mathrm{A}-\mathrm{C}$ are intended to demonstrate the impact of changing the viewing angle on the behaviour of the low frequency spectrum. $\zeta_{B}$ and $\zeta_{\text {rel }}$ are held constant in models $\mathrm{A}-\mathrm{C}$, and their ratio is set to 1 . The intrinsic synchrotron luminosity from model B is slightly higher

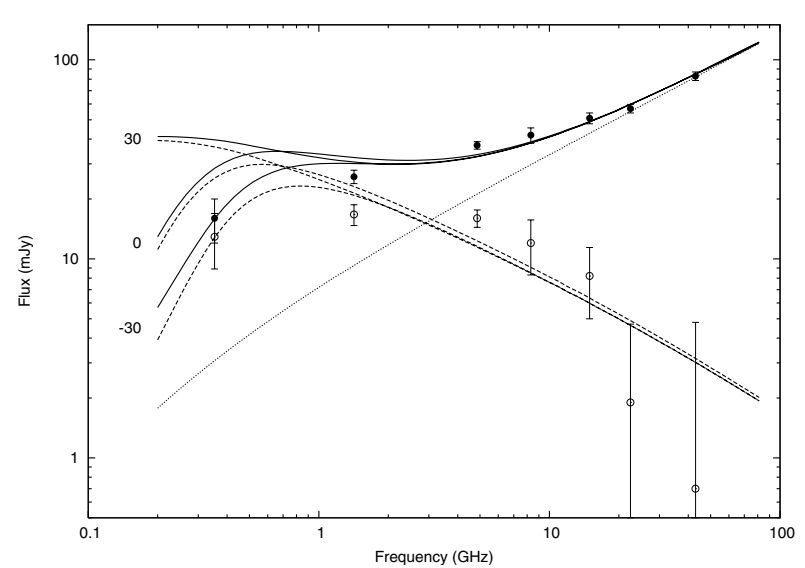

Fig. 13. Model spectra for WR 147, including total (solid), synchrotron (dashed), and thermal (dotted) flux. The observations (solid circles) are from Table 3 . The open circles are synchroton fluxes estimated by subtracting the thermal model from the total observed fluxes. Viewing angles of $-30^{\circ}$ (model A), $0^{\circ}$ (model B), and $30^{\circ}$ (model C) are shown. All models used $\eta=0.02, \gamma_{\max }=10^{5}, L_{\mathrm{OB}}=10^{5} L_{\odot}$, $\zeta=7.66 \times 10^{-3}$, and a wind filling factor, $f$, of 0.139 . The spectral index of the non-thermal electrons, $p$, was assumed to be 2 . A slight curvature to the optically thin part of the synchrotron spectrum (cf. Fig. 4) arises from IC cooling.

than that in models $\mathrm{A}$ and $\mathrm{C}$ because the stars in model $\mathrm{B}$ are closer together and this model has a viewing angle of $0^{\circ}$. The fit to the $353 \mathrm{MHz}$ data point is very sensitive to the viewing angle, and once this point is fit moderately well, the goodnessof-fit of the spectral models is largely determined by the flux computed at 1.4 and $5 \mathrm{GHz}$, given the smaller uncertainties we have derived for the observed fluxes at these two frequencies. We have calculated further models at various viewing angles, and find that we cannot obtain satisfactory fits to both the 1.4 and $5 \mathrm{GHz}$ data points with $\zeta_{B}=\zeta_{\text {rel }}$.

In an attempt to improve the spectral fit, we have calculated numerous models designed to explore the relevant parameter space. Firstly, we varied the wind momentum ratio in the range 0.02-0.012 (Pittard et al. 2002). Since the half-opening angle of the WCR is only weakly dependent on $\eta$ in this range 


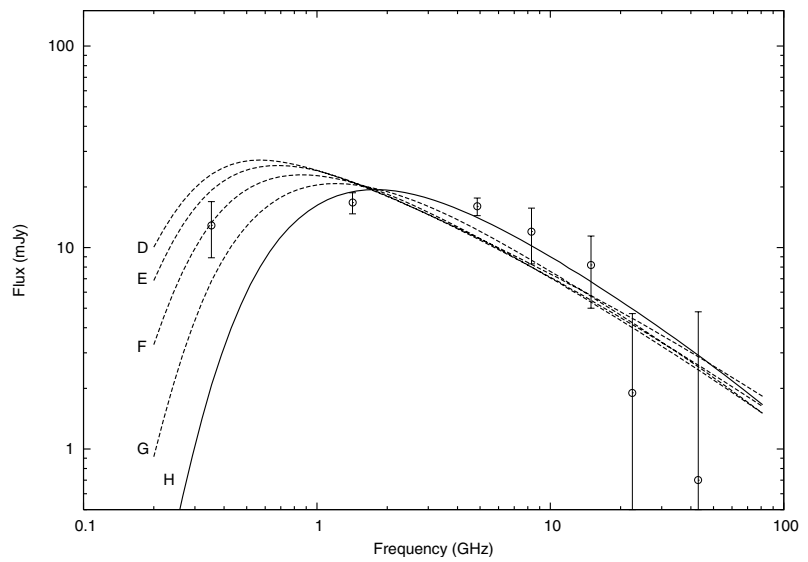

Fig. 14. Model synchrotron spectra for WR 147 at a viewing angle of $0^{\circ}$ and $\zeta_{B} / \zeta_{\text {rel }}=1,10^{-1}, 10^{-2}, 10^{-3}$ and $10^{-4}$ (models D-H respectively, with $10^{-4}$ solid - see Table 4$)$. Data points are the same as in Fig. 13.

$\left(\theta_{\text {cd }} \propto \eta^{1 / 3}\right.$, see Eichler \& Usov 1993), for a given viewing angle there is very little difference to the shape of the spectrum. The normalization of the free-free and synchrotron emission changes slightly, but this can be compensated for by adjusting the degree of clumping and the value of $\zeta$, respectively. We also performed calculations with an increased amount of clumping in the undisturbed winds, with the mass-loss rates of both stars adjusted downwards in order to obtain the desired amount of free-free emission. Changing the volume filling factor of the clumps from $f=0.139$ to $f=0.03$ produces an insignificant change in the flux at $353 \mathrm{MHz}$.

To simulate crudely a decrease in acceleration efficiency at the shocks away from the line-of-centers between the stars, we have also calculated models where the synchrotron emission and absorption is limited to a region within $\pi r_{\mathrm{O}} / 2$ of the axis of symmetry of the WCR. While this distance is somewhat arbitrary, we note that this change has only a small effect on the synchrotron spectrum once it is renormalized (by increasing $\zeta$ ).

The basic problem with all of the models noted so far is their total emission spectrum is too flat between 1.5 and $5 \mathrm{GHz}$ (see Fig. 13). The fit in this frequency range can be improved by shifting the low-frequency turnover in the model synchrotron spectrum to a higher frequency. As demonstrated in Fig. 13, fits to these two data points cannot be achieved effectively with free-free opacity alone. The turnover can be moved to a higher frequency by decreasing the magnetic energy density relative to the relativistic energy density, which increases the impact of the Razin effect (recall $\nu_{\mathrm{R}} \propto 1 / \mathrm{B}$ ). We induce this reduction in the magnetic energy density by setting $\zeta_{B} \neq \zeta_{\text {rel }}$, as described in Sect. 3.6. The resulting synchrotron spectra are shown in Fig. 14, where it is clear that the turnover due to the Razin effect moves to higher frequencies as $\zeta_{\mathrm{B}} / \zeta_{\text {rel }}$ decreases. The best fit to the 1.4 and $5 \mathrm{GHz}$ data is attained with $\zeta_{B} / \zeta_{\text {rel }}=10^{-4}$, which also gives the best fit to all other data points except that at $353 \mathrm{MHz}$, which is poor. No attempt has been made to improve the fits in models D-H by adjusting any other parameters, though $\zeta_{B}$ and $\zeta_{\text {rel }}$ were adjusted to renormalize the spectrum in order to keep the comparison simple. When we reduce $\zeta_{B}$
Table 4. Summary of parameters used in our models of WR 147 with $p=2$ (models A-I) and $p=1.4$ (models $\mathrm{J}$ and $\mathrm{K}$ ). A best fit to the data was only attempted with models $\mathrm{I}, \mathrm{J}$ and $\mathrm{K}-$ models $\mathrm{A}-\mathrm{H}$ are used to demonstrate the effect of varying the viewing angle or $\zeta_{B} / \zeta_{\text {rel }}$. All models include free-free absorption, SSA, the Razin effect, and IC cooling, and were calculated with $\eta=0.02$ and $\gamma_{\max }=10^{5}$. Models A-I had $f=0.139$ and $L_{\mathrm{OB}}=10^{5} L_{\odot}$, model $\mathrm{J}$ had $f=0.170$ and $L_{\mathrm{OB}}=10^{5} L_{\odot}$, and model $\mathrm{K}$ had $f=0.157$ and $L_{\mathrm{OB}}=4 \times 10^{5} L_{\odot}$.

\begin{tabular}{lcll}
\hline \hline Model & Viewing angle $\left(^{\circ}\right)$ & $\zeta_{\mathrm{B}} / \zeta_{\text {rel }}$ & $\zeta_{\text {rel }}$ \\
\hline $\mathrm{A}$ & -30 & 1 & $7.66 \times 10^{-3}$ \\
$\mathrm{~B}$ & 0 & 1 & $7.66 \times 10^{-3}$ \\
$\mathrm{C}$ & 30 & 1 & $7.66 \times 10^{-3}$ \\
$\mathrm{D}$ & 0 & 1 & $7.27 \times 10^{-3}$ \\
$\mathrm{E}$ & 0 & $10^{-1}$ & $2.00 \times 10^{-2}$ \\
$\mathrm{~F}$ & 0 & $10^{-2}$ & $5.69 \times 10^{-2}$ \\
$\mathrm{G}$ & 0 & $10^{-3}$ & $1.72 \times 10^{-1}$ \\
$\mathrm{H}$ & 0 & $10^{-4}$ & $5.57 \times 10^{-1}$ \\
$\mathrm{I}$ & 30 & $10^{-4}$ & $5.56 \times 10^{-1}$ \\
$\mathrm{~J}$ & -30 & 1 & $1.38 \times 10^{-2}$ \\
$\mathrm{~K}$ & -60 & 1 & $1.54 \times 10^{-2}$ \\
\hline
\end{tabular}

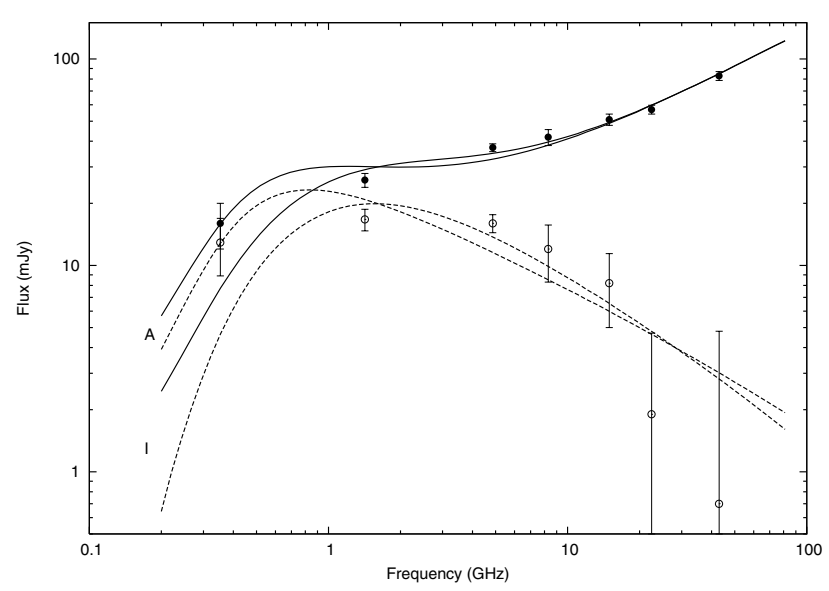

Fig. 15. The total (solid) and synchrotron (dashed) spectra obtained with model I (see Table 4), where $\zeta_{B} / \zeta_{\text {rel }}=10^{-4}$. Also shown is model A for comparison. The thermal emission model is the same as used in Fig. 13.

the synchrotron luminosity drops, and we must increase $\zeta_{\text {rel }}$ in order to maintain a specific synchrotron luminosity. This has the consequence that the value of $\zeta_{\text {rel }}$ is very close to unity in model $\mathrm{H}$.

A better fit to the $353 \mathrm{MHz}$ data point can be attained, in part, by increasing the viewing angle to reduce the impact of free-free opacity. We have attempted to fit the data by varying the viewing angle, $\zeta_{B}$ and $\zeta_{\text {rel }}$. The resulting best fit is shown in Fig. 15 (model I), and its weighted $\chi^{2}$ value is 10.7 . With 4 degrees of freedom, the reduced $\chi^{2}=2.7$. At the high frequency end the model can be made to more closely fit the data points by reducing $\gamma_{\max }$, though given the uncertainty in the data points, this has little impact on the goodness of the model fit.

A fundamental concern is the models suggest the DSA process in WR 147 is highly efficient, with an uncomfortably large fraction of the pre-shock energy transferred to the non-thermal electrons. While there are several ways in which the model 
assumptions might lead us to overestimate $\zeta_{\text {rel }}$ (e.g., the magnetic field in the WCR may not be completely tangled resulting in anisotropic emission; the thermal energy density of the WCR may have been underestimated through our choice of mass-loss rates and/or wind speeds, etc.), these possible solutions are either rather unsatisfactory in themselves, and/or unlikely to substantially increase $\zeta_{B} / \zeta_{\text {rel }}$. We note instead that in situations where the particle acceleration is highly efficient, the shock precursor (which influences the subshock compression, the injection and acceleration efficiencies, and the shape of the non-thermal particle energy spectrum) is modified by the back-pressure of energetic particles (e.g., Ellison et al. 2004). As noted in Sect. 2.2, this can create a concave curvature to the energy spectrum of the non-thermal particles, where $p$ decreases with increasing energy. Two other effects can change the overall slope of the energy spectrum. First, the softer equation of state of the relativistic particles makes the shock plasma more compressible, and increases the overall compression ratio, $r_{\text {tot }}$ (when the partial pressure from relativistic particles dominates the total pressure of the plasma, $\left.r_{\text {tot }} \rightarrow 7\right)$. Since $p=\left(r_{\text {tot }}+2\right) /\left(r_{\text {tot }}-1\right)$, a flatter power-law index results $(p=1.5$ in the limit that $\left.r_{\text {tot }}=7\right)$. Second, the overall compression ratio can become arbitrarily large as the highest energy particles escape from the shocks (see Ellison et al. 2004, for further discussion), and in this limit $p \rightarrow 1$.

Since our modelling suggests that the non-thermal particle acceleration in WR 147 is highly efficient, we have explored fits to the data where $p$ is reduced from its "standard" value of 2 . With $\zeta_{\text {rel }}=\zeta_{B}$ and the model parameters noted earlier in this section, better fits to the total flux can be obtained with $p=1.4$. The resulting fit (model J) is shown in Fig. 16. $\zeta=1.4 \times 10^{-2}$ indicates that the particle acceleration is indeed efficient, justifying our adjustment of $p$. A $p=1.4$ spectrum corresponds to an overall compression ratio of $8.5\left(r_{\text {tot }}=(p+2) /(p-1)\right)$, and is comparable to that achieved in simulations of supernova remnants with highly efficient particle acceleration (Ellison et al. 2004). The high frequency synchrotron fluxes in the model can be reduced by increasing $L_{\mathrm{OB}}$, while maintaining a similar quality of fit (model K, see Fig. 16), though we again emphasize that the fits to the WR 147 data are to the total flux, not to model deduced synchrotron fluxes which are very uncertain.

An important point is that as the overall compression increases $\left(r_{\text {tot }}>4\right)$, the subshock compression ratio declines. Since the subshock is responsible for heating the thermal gas, the post-shock temperature drops below the value expected in the strong-shock ideal-gas limit. Analysis of the X-ray data from WR 147 indicates that the temperature of peak emission from the thermal gas is $k T \approx 0.9 \mathrm{keV}$ (Skinner et al. 1999). This can be compared to the theoretical value, $k T=$ $1.96 \mu v_{8}^{2} \mathrm{keV}$, expected after thermalization in the strong-shock ideal-gas limit for material of mean particle mass $\mu m_{\mathrm{H}}$ flowing into a stationary shock at a typical wind terminal velocity $v_{\infty}=1000 v_{8} \mathrm{~km} \mathrm{~s}^{-1}$. As most of the X-ray emission should arise from the shocked WN8 wind (see Pittard \& Stevens 2002), we set $\mu=1.17$ and $v_{8}=0.95$. The expected temperature is then $k T=2.1 \mathrm{keV}$. While the contrast is not as large as that seen in WR 140, where the theoretical temperature is 5 times higher than what is observed (Pollock et al. 2005),

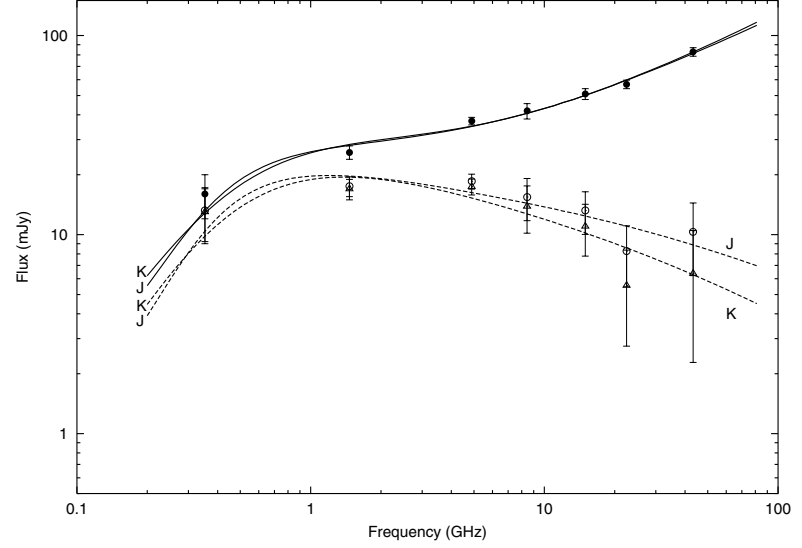

Fig. 16. The total (solid), and synchrotron (dashed) spectra for WR 147 with $p=1.4$ and $\eta=0.02$. Model $\mathrm{J}$ has $\zeta_{B}=\zeta_{\text {rel }}=1.4 \times 10^{-2}$, $f=0.17, L_{\mathrm{OB}}=10^{5} L_{\odot}$ and a viewing angle of $-30^{\circ}$. The weighted $\chi^{2}=4.97$, and the reduced $\chi^{2}=1.24$. Model $\mathrm{K}$ has $\zeta_{B}=\zeta_{\text {rel }}=$ $1.54 \times 10^{-2}, f=0.16, L_{\mathrm{OB}}=4 \times 10^{5} L_{\odot}$ and a viewing angle of $-60^{\circ}$. The weighted $\chi^{2}=5.25$, but there is only 2 degrees of freedom and the reduced $\chi^{2}=2.63$. The open circles (triangles) are synchrotron fluxes estimated by subtracting the thermal fluxes in model $\mathbf{J}$ (model K) from the total observed fluxes. The synchrotron data points are different between models $\mathrm{J}$ and $\mathrm{K}$ and from those in Figs. 13 and 14 because of the changes in the thermal flux by way of the filling factor.

we nevertheless conclude that there is perhaps some additional support for highly efficient particle acceleration.

\section{Summary and future directions}

We have extended the work in Paper I by including the evolution of the non-thermal electron energy distribution in the postshock flow. IC cooling is by far the most important process to consider, but we have also calculated the degree of ionic cooling for completeness.

We have demonstrated that the geometry of the WCR and the streamlines of the flow within it lead to a spatially dependent break frequency in the synchrotron emission. We therefore do not observe a single, sharp break in the synchrotron spectrum integrated over the WCR, but rather a steepening of the synchrotron spectrum towards higher frequencies.

As expected, IC cooling reduces the overall brightness of the synchrotron emission from the WCR, but more so near the CD than near the shocks. The synchrotron emission appears brighter at the WR shock than at the O shock when the $\mathrm{O}$ star dominates the luminosity of the system. In this situation, IC cooling has a somewhat lesser effect at the WR shock. Current radio interferometers do not have the spatial resolution and sensitivity needed to detect such spatial variation, but the predicted morphology may allow future observations to determine whether the non-thermal electrons are primarily accelerated at the shocks through the DSA scheme.

We find that the observed synchrotron emission may decrease with decreasing $D_{\text {sep }}$, through a combination of IC cooling, free-free absorption, and the Razin effect. This is consistent with the non-detection of non-thermal emission from short period CWB systems. 
We have also investigated how the synchrotron emission varies with the assumed $B$-field, and have discovered that it is very difficult for SSA to produce high turnover frequencies $(\gtrsim 1 \mathrm{GHz})$. It is much more likely that the cause of these turnovers is free-free absorption through the circumbinary envelope, or the Razin effect. If the optically thin thermal radio emission from the WCR, which scales as $D_{\text {sep }}^{-1}$, becomes comparable to the thermal emission from the unshocked winds, it is possible to obtain a composite-like spectrum entirely from thermal processes.

Furthermore, the total free-free emission from CWB systems is likely to vary with binary separation, though the exact nature of the variation depends on the degree and distribution of clumping in the system. This has important consequences for deriving mass-loss rates for stars in binary systems using radio fluxes, with the implication that they may be significantly over- or under-estimated. Our models show that it is preferable to use a high frequency $(>15 \mathrm{GHz})$ for estimates of mass-loss rates.

We have applied our new model to WR 147, and find that the introduction of IC cooling allows a better fit to the high frequency data than obtained in Paper I. However, since a weighted $\chi^{2}$ measure is biased by the relatively low uncertainity of the 1.4 and $5 \mathrm{GHz}$ data points, any model which fits these points will be preferred, and in this respect, the other data points are almost irrelevant given their relative weights. With the data currently available, we find that the best fits to the 1.4 and $5 \mathrm{GHz}$ fluxes are obtained when either the magnetic field energy density is substantially less than the non-thermal electron energy density, or when the injected non-thermal electron spectrum has a power-law index of $p=1.4$. We find that we require efficient electron acceleration in both cases, which leads us to favour the model where $p=1.4$, since non-linear calculations of DSA show a hardening of the non-thermal particle spectrum.

In summary, our analysis of the radio data from WR 147 indicates that efficient particle acceleration occurs in this system, and that the shock structure may be modified by the backpressure of energetic particles. We emphasize that our current models are in many ways very simplistic, and in order that these conclusions can be given a firmer footing, more sophisticated models which self-consistently include the back-reaction of the accelerated particles on the shock structure need to be developed. Such calculations, unfortunately, are not trivial, and a further complication is that the shock obliquity may change with off-axis distance. As the obliquity increases, the injection rates may reduce, so that some parts of the shocks may be highly modified, while other parts will be unmodified.

Such self-consistent models would also be of more general use, because despite the apparent efficiency of DSA, X-ray spectra from CWBs have been modelled and interpreted assuming that the shocks place an insignificant fraction of their energy into non-thermal particles (e.g., Stevens et al. 1996; Zhekov \& Skinner 2000; Pittard \& Corcoran 2002). These works have led to inferences for important quantities such as the mass-loss rates, wind speeds and composition, and the rate of electron and proton equilibration. However, the strong coupling between particle acceleration and thermal heating implies that the inferences made from X-ray observations may differ substantially between interpretations that include particle acceleration self-consistently and those that do not. Future work should address this issue and models should attempt to fit radio and X-ray data simultaneously. Finally, to further improve our understanding of particle acceleration in WR 147, and other colliding wind binaries, accurate and precise radiometry is required in order to distinguish between models.

Acknowledgements. We would like to thank Don Ellison, Sam Falle, Tom Hartquist, Sven Van Loo, Perry Williams, and the anonymous referee for helpful comments. J.M.P. is supported by a University Research Fellowship from the Royal Society. This research has made use of NASA's Astrophysics Data System Abstract Service.

\section{Appendix A: Synchrotron self-absorption (SSA)}

For an isotropic electron distribution function $f(p)$, the absorption coefficient for SSA is given by (see Rybicki \& Lightman 1979, Eq. (6.46)):

$\alpha_{v}=\frac{c^{2}}{8 \pi h v^{3}} \int 4 \pi p^{2} \mathrm{~d} p\left(f\left(p_{1}\right)-f(p)\right) P_{v}$

where $P_{v}$ is the magneto-bremsstrahlung emissivity and

$p_{1}^{2}=\left(\gamma^{2}-1\right)\left(m_{\mathrm{e}} c\right)^{2}+(h v / c)^{2}-2 h v \gamma m_{\mathrm{e}}$.

Using $f(p)=\frac{n(E)}{4 \pi p^{2}} \frac{\mathrm{d} E}{\mathrm{~d} p}$, we find that

$\alpha_{v}=\frac{c^{2}}{8 \pi h v^{3}} \int\left(\frac{n\left(E_{1}\right)}{p_{1} E_{1}}-\frac{n(E)}{p E}\right) P_{v} p E \mathrm{~d} E$,

where

$E_{1}=E-h v=\gamma m_{\mathrm{e}} c^{2}-h v$.

Re-writing the integral in Eq. (A.3) in terms of $\gamma$, we get

$$
\begin{aligned}
\alpha_{v}= & \frac{m_{\mathrm{e}}^{2} c^{5}}{8 \pi h \nu^{3}} \\
& \times \int\left(\frac{n\left(\gamma-\frac{h \nu}{m_{\mathrm{e}} c^{2}}\right)}{p_{1} E_{1}}-\frac{n(\gamma)}{p E}\right) P_{v} \gamma \sqrt{\gamma^{2}-1} \mathrm{~d} \gamma .
\end{aligned}
$$

We evaluate Eq. (A.5) to find the SSA for any isotropic distribution of particles $n(\gamma)$.

\section{References}

Bell, A. R. 1978, MNRAS, 182, 147

Blandford, R., \& Eichler, D. 1987, PhR, 154, 1

Blondin, J. M., \& Cioffi, D. F. 1989, ApJ, 345, 853

Blondin, J. M., \& Marks, B. S. 1996, New Astron., 1, 235

Chapman, J. M., Leitherer, C., Koribalski, B., Bouter, R., \& Storey, M. 1999, ApJ, 518, 890

Chen, W., \& White, R. L. 1991, ApJ, 366, 512

Cherepashchuk, A. M. 1990, Sov. Astr., 34, 481

Churchwell, E., Bieging, J. H., van der Hucht, K. A., et al. 1992, ApJ, 393, 329

Contreras, M. E., \& Rodríguez, L. F. 1999, ApJ, 515, 762

Contreras, M. E., Rodriguez, L. F., Gomez, Y., \& Velazquez, A. 1996, ApJ, 469, 329 
Cowie, L. L., \& McKee, C. F. 1977, ApJ, 211, 135

Crowther, P. A. 2003, in A Massive Star Odyssey: From Main Sequence to Supernova, IAU Symp., 212, 47

Dougherty, S. M., \& Williams, P. M. 2000, MNRAS, 319, 1005

Dougherty, S. M., Williams, P. M., van der Hucht, K. A., Bode, M. F., \& Davis, R. J. 1996, MNRAS, 280, 963

Dougherty, S. M., Williams, P. M., \& Pollacco, D. L. 2000, MNRAS, 316, 143

Dougherty, S. M., Pittard, J. M., Kasian, L., et al. 2003, A\&A, 409, 217

Dougherty, S. M., Beasley, A. J., Claussen, M. J., Zauderer, B. A., \& Bolingbroke, N. J. 2005, ApJ, 623, 447

Eichler, D., \& Usov, V. 1993, ApJ, 402, 271

Ellison, D. C., Decourchelle, A., \& Ballet, J. 2004, A\&A, 413, 189

Falle, S. A. E. G., \& Komissarov, S. S. 1996, MNRAS, 278, 586

Falle, S. A. E. G., Komissarov, S. S., \& Joarder, P. 1998, MNRAS, 297, 265

Ginzburg, V. L., \& Syrovatskii, S. I. 1965, ARA\&A, 3, 297

Howarth, I. D., \& Prinja, R. K. 1989, ApJS, 69, 527

Jardine, M., Allen, H. R., \& Pollock, A. M. T. 1996, A\&A, 314, 594

Jokipii, J. R. 1987, ApJ, 313, 842

Kellermann, K. I., \& Pauliny-Toth, I. I. K. 1981, ARA\&A, 19, 373

Klein, R. I., McKee, C. F., \& Colella, P. 1994, ApJ, 420, 213

Lamers, H. J. G. L. M., \& Waters, L. B. F. M. 1984, A\&A, 138, 25

Lépine, S. 1995, in Wolf-Rayet Stars: Binaries, Colliding Winds, Evolution, IAU Symp., 163, 411

Lépine, S., et al. 2000, AJ, 120, 3201

Lépine, S., Wallace, D., Shara, M. M., Moffat, A. F. J., \& Niemela, V. S. 2001, AJ, 122, 3407

Leveque, R. J. 1998, in Saas-Fee Advanced Course 27: Computational Methods for Astrophysical Fluid Flow., 1

Li, D. 2001, Nucl. Fusion, 41, 631

Mahadevan, R., Narayan, R., \& Yi, I. 1996, ApJ, 465, 327

Marchenko, S. V. 2002, in Interacting Winds from Massive Stars, ASP Conf. Ser., 260, 385

Mioduszewski, A. J., Dwarkadas, V. V., \& Ball, L. 2001, ApJ, 562, 869

Moffat, A. F. J., Drissen, L., Lamontagne, R., \& Robert, C. 1988, ApJ, 334,1038

Monnier, J. D., Greenhill, L. J., Tuthill, P. G., \& Danchi, W. C. 2002, ApJ, 566, 399

Moran, J. P., Davis, R. J., Spencer, R. E., Bode, M. F., \& Taylor, A. R. 1989, Nature, 340, 449

Morris, P. W., van der Hucht, K. A., Crowther, P. A., et al. 2000, A\&A, 353,624
Niemela, V. S., Shara, M. M., Wallace, D. J., Zurek, D. R., \& Moffat, A. F. J. 1998, AJ, 115, 2047

Pacholczyk, A. G. 1970, Radio astrophysics (San Francisco: W. H. Freeman and Company)

Perley, R. A., \& Taylor, G. B. 2003, The VLA Calibrator Manual, http://www.aco.nrao.edu/gtaylor/calib.html

Pittard, J. M., \& Corcoran, M. F. 2002, A\&A, 383, 636

Pittard, J. M., \& Stevens, I. R. 2002, A\&A, 388, L20

Pittard, J. M., Stevens, I. R., Williams, P. M., et al. 2002, A\&A, 388, 335

Pollock, A. M. T., Corcoran, M. F., Stevens, I. R., \& Williams, P. M. 2005, ApJ, 629, 482

Robert, C. 1994, Ap\&SS, 221, 137

Rodrigues, C. V., \& Magalhães, A. M. 2000, ApJ, 540, 412

Rybicki, G. B., \& Lightman, A. P. 1979, Radiative processes in astrophysics (New York: Wiley-Interscience)

Setia Gunawan, D. Y. A., de Bruyn, A. G., van der Hucht, K. A., \& Williams, P. M. 2001, A\&A, 368, 484

Skinner, S. L., Itoh, M., Nagase, F., \& Zhekov, S. A. 1999, ApJ, 524, 394

Stevens, I. R. 1995, MNRAS, 277, 163

Stevens, I. R., Blondin, J. M., \& Pollock, A. M. T. 1992, ApJ, 386, 265

Stevens, I. R., Corcoran, M. F., Willis, A. J., et al. 1996, MNRAS, 283, 589

Van Loo, S., Runacres, M. C., \& Blomme, R. 2004, A\&A, 418, 717

Van Loo, 2005, Ph.D. Thesis, Royal Observatory of Belgium

Van Loo, S., Runacres, M. C., \& Blomme, R. 2005, A\&A, 433, 313

Vishniac, E. T. 1994, ApJ, 428, 186

Wagner, A. Y., Falle, S. A. E. G., Hartquist, T. W., \& Pittard, J. M. 2005, A\&A, 430, 567

Walder, R. 1994, Some Aspects of the Computational Dynamics of Astrophysical Nebulae, Ph.D.-Thesis, ETH No. 10302, 1994

Walder, R., \& Folini, D. 2002, in Interacting Winds from Massive Stars, ASP Conf. Ser., 260, 595

Watson, S. K., Davis, R. J., Williams, P. M., \& Bode, M. F. 2002, MNRAS, 334, 631

White, R. L. 1985, ApJ, 289, 698

White, R. L., \& Becker, R. H. 1995, ApJ, 451, 352

Williams, P. M., van der Hucht, K. A., Pollock, A. M. T., et al. 1990, MNRAS, 243, 662

Williams, P. M., Dougherty, S. M., Davis, R. J., et al. 1997, MNRAS, 289,10

Wright, A. E., \& Barlow, M. J. 1975, MNRAS, 170, 41

Zhekov, S. A., \& Skinner, S. L. 2000, ApJ, 538, 808 